\title{
Contradiction-Tolerant Process Algebra with Propositional Signals
}

\author{
J.A. Bergstra and C.A. Middelburg \\ Informatics Institute, Faculty of Science, University of Amsterdam, \\ Science Park 904, 1098 XH Amsterdam, the Netherlands \\ J.A.Bergstra@uva.nl, C.A.Middelburg@uva.nl
}

\begin{abstract}
In a previous paper, an ACP-style process algebra was proposed in which propositions are used as the visible part of the state of processes and as state conditions under which processes may proceed. This process algebra, called ACPps, is built on classical propositional logic. In this paper, we present a version of ACPps built on a paraconsistent propositional logic which is essentially the same as CLuNs. There are many systems that would have to deal with self-contradictory states if no special measures were taken. For a number of these systems, it is conceivable that accepting self-contradictory states and dealing with them in a way based on a paraconsistent logic is an alternative to taking special measures. The presented version of ACPps can be suited for the description and analysis of systems that deal with self-contradictory states in a way based on the above-mentioned paraconsistent logic.
\end{abstract}

Keywords: process algebra, propositional signal, propositional condition, paraconsistent logic.

1998 ACM Computing Classification: D.2.1, D.2.4, F.3.1, F.4.1.

\section{Introduction}

Algebraic theories of processes such as ACP [11, CCS [27], and CSP [25], as well as most algebraic theories of processes in the style of these ones, are concerned with the behaviour of processes only. That is, the state of processes is kept invisible. In [8], an ACP-style process algebra, called ACPps, was proposed in which processes have their state to some extent visible. The visible part of the state of a process, called the signal emitted by the process, is a proposition of classical propositional logic. Propositions are not only used as signals emitted by processes, but also as conditions under which processes may proceed. The intuition is that the signal emitted by a process is a proposition that holds at its start and the condition under which processes may proceed is a proposition that must hold at its start. Thus, by the introduction of signal emitting processes, an answer is given to the question what determines whether a condition under which a process may proceed is met.

If the signals emitted by two processes are contradictory, then the signal emitted by the parallel composition of these processes is self-contradictory. For 
example, if the signals emitted by the two processes, being propositions, are each others negation, then they are contradictory and their conjunction, which is the signal emitted by the parallel composition of these processes, is selfcontradictory. Intuitively, a process emitting a self-contradictory signal is an impossibility. Therefore, a special process has been introduced in ACPps to deal with it. In practice, there are many systems that would have to deal with selfcontradictory states if no special prevention measures or special detection and resolution measures were taken. Some typical examples are web-service-oriented applications and autonomous robotic agents (see e.g. 23129130]). At least for a number of these systems, it is conceivable that accepting self-contradictory states and dealing with them in a way based on a suitable paraconsistent logic is an alternative to taking special measures. It may even be the only workable alternative because a system may have to cope with inconsistencies occurring on a large scale.

What exactly does it mean to deal with self-contradictory states in a way based on a paraconsistent logic? The systems referred to above are systems whose behaviour is made up of discrete steps where, upon each step performed, the way in which the behaviour proceeds is conditional on the current state of the system concerned. If the propositions by which the visible part of the possible states of a system can be characterized are used as conditions, then it can be established in accordance with a paraconsistent propositional logic whether a condition is met in a state. This is what is meant by dealing with self-contradictory states in a way based on a paraconsistent logic. We think that a version of the process algebra ACPps that is built on an appropriate paraconsistent propositional logic instead of classical propositional logic can be suited for the description and analysis of systems that deal with self-contradictory states in a way based on a paraconsistent logic. The important point here is that, in such a logic, it is generally not possible to deduce an arbitrary formula from two contradictory formulas.

The question remains: what is an appropriate paraconsistent propositional logic? The ones that have been proposed differ in many ways and whether one of them is more appropriate than another is fairly difficult to make out. A paraconsistent propositional logic is a logic that does not have the property that every proposition is a logical consequence of every set of hypotheses that contains contradictory propositions. A paraconsistent propositional logic with the property that every proposition is a logical consequence of every set of hypotheses that contains contradictory propositions but one is far from appropriate. Such a logic is a minimal paraconsistent logic. Maximal paraconsistency, i.e. a logical consequence relation that cannot be extended without loosing paraconsistency, is generally considered an important property. There are various other properties that have been proposed as characteristic of reasonable paraconsistent propositional logics, but their importance remains to some extent open to question.

The properties that have been proposed as characteristic of reasonable paraconsistent propositional logics do not include all properties that are required of an appropriate one to build a version of ACPps on. These properties include, 
among other things, properties needed to retain the basic axioms of ACP-style process algebras. In this paper, we present a version of ACPps built on the paraconsistent propositional logic for which the name $\mathrm{LP}^{\supset}{ }^{\mathrm{T}} \mathrm{F}$ was coined in 26 . This logic, which is essentially the same as J3 20, CLuNs [12, and LFI1 [19], has virtually all properties that have been proposed as characteristic of reasonable paraconsistent propositional logics as well as all properties that are required of an appropriate one to build a version of $\mathrm{ACPps}$ on. $\mathrm{LP}^{\supset, \mathrm{F}}$ can be replaced by any paraconsistent propositional logics with the latter properties, but among the paraconsistent propositional logics with the former properties, $\mathrm{LP}^{\supset, \mathrm{F}}$ is the only one with the latter properties.

The structure of this paper is as follows. First, we give a survey of the paraconsistent propositional logic $\mathrm{LP}^{\mathrm{D}, \mathrm{F}}$ (Section 2). Next, we present $\mathrm{BPA}_{\mathrm{ps}}^{\mathrm{ct}}$, the subtheory of the version of ACPps built on LP ${ }^{\supset, F}$ that does not support parallelism and communication (Sections 3 and 4). After that, we present $\mathrm{ACP}_{\mathrm{ps}}^{\mathrm{ct}}$, the version of $\mathrm{ACP} p$ built on $\mathrm{LP}^{\mathrm{D}, \mathrm{F}}$, as an extension of $\mathrm{BPA}_{\mathrm{ps}}^{\mathrm{ct}}$ (Sections 5 and 6). Following this, we introduce a useful additional feature, namely a generalization of the state operators from [6] (Section 7). Then, we treat the addition of guarded recursion to $\mathrm{ACP}_{\mathrm{ps}}^{\mathrm{ct}}$ (Section 8). Finally, we make some concluding remarks (Section 9).

\section{The Paraconsistent Logic $\mathrm{LP}^{\supset}, \mathrm{F}$}

A set of propositions $\Gamma$ is contradictory if there exists a proposition $A$ such that both $A$ and $\neg A$ can be deduced from $\Gamma$. A proposition $A$ is called selfcontradictory if $\{A\}$ is contradictory. In classical propositional logic, every proposition can be deduced from a contradictory set of propositions. A paraconsistent propositional logic is a propositional logic in which not every proposition can be deduced from each contradictory set of propositions.

In 28, Priest proposed the paraconsistent propositional logic LP (Logic of Paradox). The logic introduced in this section is LP enriched with an implication connective for which the standard deduction theorem holds and a falsity constant. This logic, called $\mathrm{LP}^{\mathrm{J}, \mathrm{F}}$, is in fact the propositional fragment of CLuNs [12] without bi-implications.

$\mathrm{LP}^{\supset, \mathrm{F}}$ has the following logical constants and connectives: a falsity constant $F$, a unary negation connective $\neg$, a binary conjunction connective $\wedge$, a binary disjunction connective $\vee$, and a binary implication connective $\supset$. Truth and biimplication are defined as abbreviations: T stands for $\neg \mathrm{F}$ and $A \equiv B$ stands for $(A \supset B) \wedge(B \supset A)$.

A Hilbert-style formulation of $\mathrm{LP}^{\supset}, \mathrm{F}$ is given in Table 1 In this formulation, which is taken from [4, $A, B$, and $C$ are used as meta-variables ranging over all formulas of $\mathrm{LP}^{\supset}, \mathrm{F}$. The axiom schemas on the left-hand side of Table 1 and the single inference rule (modus ponens) constitute a Hilbert-style formulation of the positive fragment of classical propositional logic. The first four axiom schemas on the right-hand side of Table 1 allow for the negation connective to be moved inward. The fifth axiom schema on the right-hand side of Table 1 is the law of 
Table 1. Hilbert-style formulation of $\mathrm{LP}^{\supset, \mathrm{F}}$

\begin{tabular}{ll}
\hline Axiom Schemas : & \\
$A \supset(B \supset A)$ & $\neg \neg A \equiv A$ \\
$(A \supset(B \supset C)) \supset((A \supset B) \supset(A \supset C))$ & $\neg(A \supset B) \equiv A \wedge \neg B$ \\
$((A \supset B) \supset A) \supset A$ & $\neg(A \wedge B) \equiv \neg A \vee \neg B$ \\
$\mathrm{~F} \supset A$ & $\neg(A \vee B) \equiv \neg A \wedge \neg B$ \\
$(A \wedge B) \supset A$ & \\
$(A \wedge B) \supset B$ & $A \vee \neg A$ \\
$A \supset(B \supset(A \wedge B))$ & Rule of Inference : \\
$A \supset(A \vee B)$ & $\frac{A \quad A \supset B}{B}$ \\
$B \supset(A \vee B)$ & \\
$(A \supset C) \supset((B \supset C) \supset((A \vee B) \supset C))$ &
\end{tabular}

the excluded middle. This axiom schema can be thought of as saying that, for every proposition, the proposition or its negation is true, while leaving open the possibility that both are true. If we add the axiom schema $\neg A \supset(A \supset B)$, which says that any proposition follows from a contradiction, to the given Hilbertstyle formulation of $\mathrm{LP}^{\supset, \mathrm{F}}$, then we get a Hilbert-style formulation of classical propositional logic (see e.g. 4]). We write $\vdash$ for the syntactic logical consequence relation induced by the axiom schemas and inference rule of $\mathrm{LP}^{\supset}, \mathrm{F}$.

The following outline of the semantics of $\mathrm{LP}^{\supset}, \mathrm{F}$ is based on 44. Like in the case of classical propositional logic, meanings are assigned to the formulas of LP $\supset, \mathrm{F}$ by means of valuations. However, in addition to the two classical truth values $t$ (true) and $f$ (false), a third meaning $b$ (both true and false) may be assigned. A valuation for $\mathrm{LP}^{\supset, \mathrm{F}}$ is a function $\nu$ from the set of all formulas of $\mathrm{LP}^{\supset, \mathrm{F}}$ to the set $\{\mathrm{t}, \mathrm{f}, \mathrm{b}\}$ such that for all formulas $A$ and $B$ of $\mathrm{LP}^{\supset, \mathrm{F}}$ :

$$
\begin{gathered}
\nu(\mathrm{F})=\mathrm{f}, \\
\nu(\neg A)= \begin{cases}\mathrm{t} & \text { if } \nu(A)=\mathrm{f} \\
\mathrm{f} & \text { if } \nu(A)=\mathrm{t} \\
\mathrm{b} & \text { otherwise, }\end{cases} \\
\nu(A \wedge B)= \begin{cases}\mathrm{t} & \text { if } \nu(A)=\mathrm{t} \text { and } \nu(B)=\mathrm{t} \\
\mathrm{f} & \text { if } \nu(A)=\mathrm{f} \text { or } \nu(B)=\mathrm{f} \\
\mathrm{b} & \text { otherwise, }\end{cases} \\
\nu(A \vee B)= \begin{cases}\mathrm{t} & \text { if } \nu(A)=\mathrm{t} \text { or } \nu(B)=\mathrm{t} \\
\mathrm{f} & \text { if } \nu(A)=\mathrm{f} \text { and } \nu(B)=\mathrm{f} \\
\mathrm{b} & \text { otherwise, }\end{cases} \\
\nu(A \supset B)= \begin{cases}\mathrm{t} & \text { if } \nu(A)=\mathrm{f} \\
\nu(B) & \text { otherwise. }\end{cases}
\end{gathered}
$$

The classical truth-conditions and falsehood-conditions for the logical connectives are retained. Except for implications, a formula is classified as both-true- 
and-false exactly when when it cannot be classified as true or false by the classical truth-conditions and falsehood-conditions. The definition of a valuation given above shows that the logical connectives of $\mathrm{LP}^{\supset}, \mathrm{F}$ are (three-valued) truthfunctional, which means that each $n$-ary connective represents a function from $\{\mathrm{t}, \mathrm{f}, \mathrm{b}\}^{n}$ to $\{\mathrm{t}, \mathrm{f}, \mathrm{b}\}$.

For $\mathrm{LP}^{\supset}, \mathrm{F}$, the semantic logical consequence relation, denoted by $\vDash$, is based on the idea that a valuation $\nu$ satisfies a formula $A$ if $\nu(A) \in\{\mathrm{t}, \mathrm{b}\}$. It is defined as follows: $\Gamma \vDash A$ iff for every valuation $\nu$, either $\nu\left(A^{\prime}\right)=\mathrm{f}$ for some $A^{\prime} \in \Gamma$ or $\nu(A) \in\{\mathrm{t}, \mathrm{b}\}$. We have that the Hilbert-style formulation of $\mathrm{LP}^{\supset}, \mathrm{F}$ is strongly complete with respect to its semantics, i.e. $\Gamma \vdash A$ iff $\Gamma \vDash A$ (see e.g. [12]).

For all formulas $A$ of $\mathrm{LP}^{\supset}, \mathrm{F}$ in which $\mathrm{F}$ does not occur, for all formulas $B$ of $\mathrm{LP}^{\supset, \mathrm{F}}$ in which no propositional variable occurs that occurs in $A,\{A, \neg A\} \forall B$ if $\forall B$ (see e.g. [1] 1$]$ Hence, $\mathrm{LP}^{\supset}, \mathrm{F}$ is a paraconsistent propositional logic.

For $\mathrm{LP}^{\supset, \mathrm{F}}$, the logical equivalence relation $\Leftrightarrow$ is defined as for classical propositional logic: $A \Leftrightarrow B$ iff for every valuation $\nu, \nu(A)=\nu(B)$. Unlike in classical propositional logic, we do not have that $A \Leftrightarrow B$ iff $\vdash A \equiv B$.

For LP ${ }^{\supset}, \mathrm{F}$, the consistency property is defined as to be expected: $A$ is consistent iff for every valuation $\nu, \nu(A) \neq \mathrm{b}$.

The following are some important properties of $\mathrm{LP}^{\supset, \mathrm{F}}$ :

(a) containment in classical logic: $\vdash \subseteq \vdash_{\mathrm{CL}}$

(b) proper basic connectives: for all sets $\Gamma$ of formulas of $\mathrm{LP}^{\supset, \mathrm{F}}$ and all formulas $A, B$, and $C$ of $\mathrm{LP}^{\supset}, \mathrm{F}$ :

(b $\left.\mathrm{b}_{1}\right) \Gamma \cup\{A\} \vdash B \quad$ iff $\Gamma \vdash A \supset B$,

$\left(\mathrm{b}_{2}\right) \Gamma \vdash A \wedge B \quad$ iff $\Gamma \vdash A$ and $\Gamma \vdash B$,

(b) $\Gamma \cup\{A \vee B\} \vdash C$ iff $\Gamma \cup\{A\} \vdash C$ and $\Gamma \cup\{B\} \vdash C$;

(c) weakly maximal paraconsistency relative to classical logic: for all formulas $A$ of $\mathrm{LP}^{\supset}$, $\mathrm{F}$ with $\forall A$ and $\vdash_{\mathrm{CL}} A$, for the minimal consequence relation $\vdash^{\prime}$ such that $\vdash \subseteq \vdash^{\prime}$ and $\vdash^{\prime} A$, for all formulas $B$ of $\mathrm{LP}^{\supset, \mathrm{F}}, \vdash^{\prime} B$ iff $\vdash_{\mathrm{CL}} B$;

(d) strongly maximal absolute paraconsistency: for all logics $\mathcal{L}$ with the same logical constants and connectives as $\mathrm{LP}^{\mathrm{D}}, \mathrm{F}$ and a consequence relation $\vdash^{\prime}$ such that $\vdash \subset \vdash^{\prime}, \mathcal{L}$ is not paraconsistent;

(e) internalized notion of consistency: $A$ is consistent iff $\vdash(A \supset \mathrm{F}) \vee(\neg A \supset \mathrm{F})$;

(f) internalized notion of logical equivalence: $A \Leftrightarrow B$ iff $\vdash(A \equiv B) \wedge(\neg A \equiv \neg B)$;

(g) the laws given in Table 2 hold for the logical equivalence relation of $\mathrm{LP}^{\supset, \mathrm{F}}$.

Properties (a)-(f) have been mentioned relatively often in the literature (see e.g. [1/2/3/5[12 19]). Properties (a), ( $\left.b_{1}\right),(c)$, and (d) make LP ${ }^{\supset, F}$ an ideal paraconsistent logic in the sense made precise in 2 . By property $(\mathrm{e}), \mathrm{LP}^{\supset}, \mathrm{F}$ is also a logic of formal inconsistency in the sense made precise in [19]. Properties (a)-(c) indicate that $\mathrm{LP}^{\supset}, \mathrm{F}$ retains much of classical propositional logic. Actually, property (c) can be strengthened to the following property: for all formulas $A$ of $\mathrm{LP}^{\supset, \mathrm{F}}, \vdash A$ iff $\vdash_{\mathrm{CL}} A$.

\footnotetext{
${ }^{1}$ We use the notation $\vdash A$ for $\emptyset \vdash A, \forall A$ for not $\emptyset \vdash A$, and $\Gamma \nvdash A$ for not $\Gamma \vdash A$.

${ }^{2}$ We use the symbol $\vdash_{\mathrm{CL}}$ to denote the logical consequence relation of classical propositional logic.
} 
Table 2. Laws that hold for the logical equivalence relation of $\mathrm{LP}^{\supset}, \mathrm{F}$

\begin{tabular}{ll}
\hline$(1) A \wedge \mathrm{F} \Leftrightarrow \mathrm{F}$ & (2) $A \vee \mathrm{T} \Leftrightarrow \mathrm{T}$ \\
$(3) A \wedge \mathrm{T} \Leftrightarrow A$ & (4) $A \vee \mathrm{F} \Leftrightarrow A$ \\
(5) $A \wedge A \Leftrightarrow A$ & (6) $A \vee A \Leftrightarrow A$ \\
(7) $A \wedge B \Leftrightarrow B \wedge A$ & (8) $A \vee B \Leftrightarrow B \vee A$ \\
$(9)(A \wedge B) \wedge C \Leftrightarrow A \wedge(B \wedge C)$ & (10) $(A \vee B) \vee C \Leftrightarrow A \vee(B \vee C)$ \\
$(11) A \wedge(B \vee C) \Leftrightarrow(A \wedge B) \vee(A \wedge C)$ & $(12) A \vee(B \wedge C) \Leftrightarrow(A \vee B) \wedge(A \vee C)$ \\
$(13)(A \supset B) \wedge(A \supset C) \Leftrightarrow A \supset(B \wedge C)$ & $(14)(A \supset C) \wedge(B \supset C) \Leftrightarrow(A \vee B) \supset C$ \\
$(15)(A \vee \neg A) \supset B \Leftrightarrow B$ & $(16) A \supset(B \supset C) \Leftrightarrow(A \wedge B) \supset C$ \\
\hline
\end{tabular}

From Theorem 4.42 in $[1$, we know that there are exactly 8192 different threevalued paraconsistent propositional logics with properties (a) and (b). From Theorem 2 in 2, we know that properties (c) and (d) are common properties of all three-valued paraconsistent propositional logics with properties $(\mathrm{a})$ and $\left(\mathrm{b}_{1}\right)$. From Fact 103 in [19, we know that property (f) is a common property of all three-valued paraconsistent propositional logics with properties (a), (b) and (e). Moreover, it is easy to see that that property (e) is a common property of all three-valued paraconsistent propositional logics with properties (a) and (b). Hence, each three-valued paraconsistent propositional logic with properties (a) and (b) has properties (c)-(f) as well.

Property $(\mathrm{g})$ is not a common property of all three-valued paraconsistent propositional logics with properties (a) and (b). To our knowledge, properties like property (g) are not mentioned in the literature. However, like property (f), property $(\mathrm{g})$ is essential for the process algebra presented in this paper. Among the 8192 three-valued paraconsistent propositional logics with properties (a)-(e), which are considered desirable properties, $\mathrm{LP}^{\supset}, \mathrm{F}$ is one out of four with the essential properties (f) and (g).

Proposition 1 (Almost Uniqueness). There are exactly four three-valued paraconsistent propositional logics with the logical constants and connectives of $L P^{\supset}, \mathrm{F}$ that have the properties (a)-(g) mentioned above.

Proof. Because property (f) is a common property of all 8192 three-valued paraconsistent propositional logics with properties (a)-(e), it is sufficient to prove that, among these 8192 logics, there exists only one that has property $(\mathrm{g})$. Because 'non-deterministic truth tables' that uniquely characterize the 8192 logics are given in [2], the theorem can be proved by showing that, for each of the connectives, only one of the ordinary truth tables represented by the nondeterministic truth table for that connective is compatible with the laws given in Table 2. It can be shown by short routine case analyses that only one of the 8 ordinary truth tables represented by the non-deterministic truth tables for conjunction is compatible with laws (1), (3), (5), and (7) and only one of the 32 ordinary truth tables represented by the non-deterministic truth tables for 
disjunction is compatible with laws (2), (4), (6), and (8). The truth tables concerned are compatible with laws (9)-(12) as well. Given the ordinary truth table for conjunction and disjunction so obtained, it can be shown by slightly longer routine case analyses that exactly four of the 16 ordinary truth tables represented by the non-deterministic truth table for implication are compatible with laws (13)-(15). The four truth tables concerned are compatible with law (16) as well.

The next corollary follows from the proof of Proposition 1.

Corollary 1 (Uniqueness). $L P^{\supset, \mathrm{F}}$ is the only three-valued paraconsistent propositional logic with the logical constants and connectives of $L P^{\supset}, \mathrm{F}$ that has the properties $(a)-(g)$ mentioned above and moreover the property that the law $\neg \neg A \Leftrightarrow A$ holds for its logical equivalence relation.

Corollary 1 may be of independent importance to the area of paraconsistent logics.

From now on, we will use the following abbreviations: $A \leftrightarrow B$ stands for $(A \equiv B) \wedge(\neg A \equiv \neg B)$ and $\circ A$ stands for $(A \supset \mathrm{F}) \vee(\neg A \supset \mathrm{F})$.

In Section 3, where we will use formulas of $\mathrm{LP}^{\supset, \mathrm{F}}$ as terms, equality of formulas will be interpreted as logical equivalence. This means that equality of formulas can be formally proved using the fact that $A \Leftrightarrow B$ iff $\vdash A \leftrightarrow B$. This fact also suggests that $\mathrm{LP}^{\supset}, \mathrm{F}$ may be Blok-Pigozzi algebraizable [18. It is shown in [19] that actually all 8192 three-valued paraconsistent propositional logics referred to above are Blok-Pigozzi algebraizable. Although there must exist one, a conditional-equational axiomatization of the algebras concerned in the case of $\mathrm{LP}^{\supset}, \mathrm{F}$ has not yet been devised. Owing to this, the equations derivable in the version of ACPps built on $\mathrm{LP}^{\supset}, \mathrm{F}$ presented in this paper cannot always be derived by equational reasoning only.

\section{Contradiction-Tolerant BPA with Propositional Signals}

BPAps is a subtheory of ACPps that does not support parallelism and communication. In this section, we present the contradiction-tolerant version of BPAps. In this version, which is called $\mathrm{BPA}_{\mathrm{ps}}^{\mathrm{ct}}$, processes have their state to some extent visible. The visible part of the state of a process, called the signal emitted by the process, is a proposition of $\mathrm{LP}^{\supset, \mathrm{F}}$. These propositions are not only used as signals emitted by processes, but also as conditions under which processes may proceed. The intuition is that the signal emitted by a process is a proposition that holds at its start and the condition under which processes may proceed is a proposition that must holds at its start.

In $\mathrm{BPA}_{\mathrm{ps}}^{\mathrm{ct}}$, just as in BPAps, it is assumed that a fixed but arbitrary finite set $\mathrm{A}$ of actions, with $\delta \notin \mathrm{A}$, and a fixed but arbitrary finite set $\mathrm{B}_{\text {at }}$ of atomic propositions have been given. We write $\mathrm{A}_{\delta}$ for $\mathrm{A} \cup\{\delta\}$.

The algebraic theory $\mathrm{BPA}_{\mathrm{ps}}^{\mathrm{ct}}$ has two sorts:

- the sort $\mathbf{P}$ of processes;

- the sort $\mathbf{B}$ of propositions. 
The algebraic theory $\mathrm{BPA}_{\mathrm{ps}}^{\mathrm{ct}}$ has the following constants and operators to build terms of sort $\mathbf{B}$ :

- for each $P \in \mathrm{B}_{\mathrm{at}}$, the atomic proposition constant $P: \mathbf{B}$;

- the falsity constant $\mathrm{F}: \mathbf{B}$;

- the unary negation operator $\neg: \mathbf{B} \rightarrow \mathbf{B}$;

- the binary conjunction operator $\wedge: \mathbf{B} \times \mathbf{B} \rightarrow \mathbf{B}$;

- the binary disjunction operator $\vee: \mathbf{B} \times \mathbf{B} \rightarrow \mathbf{B}$;

- the binary implication operator $\supset: \mathbf{B} \times \mathbf{B} \rightarrow \mathbf{B}$.

The algebraic theory $\mathrm{BPA}_{\mathrm{ps}}^{\mathrm{ct}}$ has the following constants and operators to build terms of sort $\mathbf{P}$ :

- the deadlock constant $\delta: \mathbf{P}$

- for each $a \in \mathrm{A}$, the action constant $a: \mathbf{P}$;

- the inaccessible process constant $\perp: \mathbf{P}$;

- the binary alternative composition operator $+: \mathbf{P} \times \mathbf{P} \rightarrow \mathbf{P}$;

- the binary sequential composition operator $\cdot: \mathbf{P} \times \mathbf{P} \rightarrow \mathbf{P}$;

- the binary guarded command operator $: \rightarrow: \mathbf{B} \times \mathbf{P} \rightarrow \mathbf{P}$;

- the binary signal emission operator $\mathbf{\wedge}: \mathbf{B} \times \mathbf{P} \rightarrow \mathbf{P}$.

It is assumed that there are infinitely many variables of sort $\mathbf{P}$, including $x, y$, and $z$.

We use infix notation for the binary operators. The following precedence conventions are used to reduce the need for parentheses. The operators to build terms of sort $\mathbf{B}$ bind stronger than the operators to build terms of sort $\mathbf{P}$. The operator - binds stronger than all other binary operators to build terms of sort $\mathbf{P}$ and the operator + binds weaker than all other binary operators to build terms of sort $\mathbf{P}$.

Let $p$ and $q$ be closed terms of sort $\mathbf{P}$ and $\phi$ be a closed term of sort $\mathbf{B}$. Intuitively, the constants and operators to build terms of sort $\mathbf{P}$ can be explained as follows:

- $\delta$ is not capable of doing anything, the proposition that holds at the start of $\delta$ is $\mathrm{T}$;

- $a$ is only capable of performing action $a$ unconditionally and next terminating successfully, the proposition that holds at the start of $a$ is T;

$-\perp$ is not capable of doing anything; there is an inconsistency at the start of $\perp$;

$-p+q$ behaves either as $p$ or as $q$ but not both, the proposition that holds at the start of $p+q$ is the conjunction of the propositions that hold at the start of $p$ and $q$;

$-p \cdot q$ first behaves as $p$ and on successful termination of $p$ it next behaves as $q$, the proposition that holds at the start of $p \cdot q$ is the proposition that holds at the start of $p$;

$-\phi: \rightarrow p$ behaves as $p$ under condition $\phi$, the proposition that holds at the start of $\phi: \rightarrow p$ is the implication with $\phi$ as antecedent and the proposition that holds at the start of $p$ as consequent; 
Table 3. Axioms of $\mathrm{BPA}_{\mathrm{ps}}^{\mathrm{ct}}$

\begin{tabular}{|c|c|c|c|}
\hline$x+y=y+x$ & $\mathrm{~A} 1$ & $x+\perp=\perp$ & NE1 \\
\hline$(x+y)+z=x+(y+z)$ & $\mathrm{A} 2$ & $\perp \cdot x=\perp$ & NE2 \\
\hline$x+x=x$ & A3 & $a \cdot \perp=\delta$ & NE3 \\
\hline$(x+y) \cdot z=x \cdot z+y \cdot z$ & A4 & & \\
\hline$(x \cdot y) \cdot z=x \cdot(y \cdot z)$ & A5 & & \\
\hline$x+\delta=x$ & $\mathrm{~A} 6$ & & \\
\hline$\delta \cdot x=\delta$ & A7 & if $\vdash \phi \leftrightarrow \psi$ & IMP \\
\hline $\mathrm{\top}: \rightarrow x=x$ & GC1 & $\mathrm{\top} \wedge x=x$ & SE1 \\
\hline $\mathrm{F}: \rightarrow x=\delta$ & $\mathrm{GC} 2$ & $\mathrm{~F} \wedge x=\perp$ & SE2 \\
\hline$\phi: \rightarrow \delta=\delta$ & GC3 & $\phi^{\wedge} \perp=\perp$ & SE3 \\
\hline$\phi: \rightarrow(x+y)=\phi: \rightarrow x+\phi: \rightarrow y$ & $\mathrm{GC} 4$ & $\phi \wedge x+y=\phi \wedge(x+y)$ & $\mathrm{SE} 4$ \\
\hline$\phi: \rightarrow x \cdot y=(\phi: \rightarrow x) \cdot y$ & GC5 & $(\phi \wedge x) \cdot y=\phi \wedge x \cdot y$ & SE5 \\
\hline$\phi: \rightarrow(\psi: \rightarrow x)=(\phi \wedge \psi): \rightarrow x$ & GC6 & $\phi \wedge(\psi \wedge x)=(\phi \wedge \psi) \wedge x$ & SE6 \\
\hline$(\phi \vee \psi): \rightarrow x=\phi: \rightarrow x+\psi: \rightarrow x$ & $\mathrm{GC} 7$ & $\begin{array}{l}\phi \wedge(\phi: \rightarrow x)=\phi \wedge x \\
\phi: \rightarrow(\psi \wedge x)=(\phi \supset \psi) \wedge(\phi: \rightarrow x)\end{array}$ & $\begin{array}{l}\text { SE7 } \\
\text { SE8 }\end{array}$ \\
\hline
\end{tabular}

$-\phi^{\wedge} p$ behaves as $p$ if the proposition that holds at its start does not equal $\mathrm{F}$ and as $\perp$ otherwise, in the former case, the proposition that holds at the start of $\phi \wedge p$ is the conjunction of $\phi$ and the proposition that holds at the start of $p$.

The axioms of $\mathrm{BPA}_{\mathrm{ps}}^{\mathrm{ct}}$ are the axioms given in Table 3, In this table, $a$ stands for an arbitrary constant from $\mathrm{A} \cup\{\delta\}, \phi$ and $\psi$ stand for arbitrary closed terms of sort $\mathbf{B}$, and $\vdash$ is the logical consequence relation of $\mathrm{LP}^{\supset}, \mathrm{F}$. A1-A7 are the axioms of $\mathrm{BPA}_{\delta}$, the subtheory of $\mathrm{ACP}$ that does not support parallelism and communication (see e.g. [1]). NE1-NE3, GC1-GC7, and SE1-SE8 have been taken from [8], using a different numbering 3 By IMP, the axioms of $\mathrm{BPA}_{\mathrm{ps}}^{\mathrm{ct}}$ include all equations $\phi=\psi$ for which $\phi \leftrightarrow \psi$ is a theorem of $\mathrm{LP}^{\supset}, \mathrm{F}$. This is harmless because the connective $\leftrightarrow$, which is the internalization of the logical equivalence relation $\Leftrightarrow$ of $\mathrm{LP}^{\supset}, \mathrm{F}$, is a congruence.

The following generalizations of axioms SE4 and SE7 are among the equations derivable from the axioms of $\mathrm{BPA}_{\mathrm{ps}}^{\mathrm{ct}}$ :

$$
\begin{aligned}
& \phi \wedge x+\psi \wedge y=(\phi \wedge \psi) \wedge(x+y), \\
& (\phi \wedge \psi) \wedge(\phi: \rightarrow x)=(\phi \wedge \psi) \wedge x, \\
& \phi \wedge((\phi \wedge \psi): \rightarrow x)=\phi \wedge(\psi: \rightarrow x) ;
\end{aligned}
$$

\footnotetext{
3 The axioms of $\mathrm{BPA}_{\mathrm{ps}}^{\mathrm{ct}}$ are not independent: A3, A6, and A7 are derivable from GC1GC7 and IMP, NE1 and NE2 are derivable from SE1-SE8, and SE3 is derivable from SE6 and IMP.
} 
the following specialization of axiom SE4 is among the equations derivable from the axioms of $\mathrm{BPA}_{\mathrm{ps}}^{\mathrm{ct}}$ :

$$
\phi \wedge \delta+x=\phi^{\wedge} x
$$

and the following equations concerning the inaccessible process are among the equations derivable from the axioms of $\mathrm{BPA}_{\mathrm{ps}}^{\mathrm{ct}}$ :

$$
\begin{aligned}
& \phi \wedge \perp=\perp, \\
& \phi: \rightarrow \perp=(\phi \supset \mathrm{F}) \wedge \delta .
\end{aligned}
$$

The derivable equations mentioned above are derivable from the axioms of BPAps as well. The equation $\phi: \rightarrow \perp=\neg \phi \wedge \delta$, which is derivable from the axioms of BPAps, is not derivable from the axioms of $\mathrm{BPA}_{\mathrm{ps}}^{\mathrm{ct}}$.

Let $\phi$ be a closed term of sort $\mathbf{B}$ such that not $\vdash \phi \leftrightarrow \mathrm{F}$ and not $\vdash \neg \phi \leftrightarrow \mathrm{F}$. Then, because not $\vdash \phi \wedge \neg \phi \leftrightarrow \mathrm{F}$, we have that $a \cdot(\phi \wedge x+\neg \phi \wedge y)=a \cdot(\mathrm{F} \wedge$ $(x+y))=\delta$, which is derivable from the axioms of BPAps, is not derivable from the axioms of $\mathrm{BPA}_{\mathrm{ps}}^{\mathrm{ct}}$. This shows the main difference between $\mathrm{BPA}_{\mathrm{ps}}^{\mathrm{ct}}$ and BPAps: the alternative composition of two processes of which the propositions that hold at the start of them are contradictory does not lead to an inconsistency in $\mathrm{BPA}_{\mathrm{ps}}^{\mathrm{ct}}$, whereas it does lead to an inconsistency in BPAps. This is why BPA $\mathrm{ps}_{\mathrm{ps}}^{\mathrm{ct}}$ is called the contradiction-tolerant version of BPAps.

Let $\phi$ be a closed term of sort $\mathbf{B}$ such that not $\vdash \phi \leftrightarrow \mathrm{F}$ and not $\vdash \neg \phi \leftrightarrow \mathrm{F}$. We can derive $a \cdot(\phi \wedge b+\neg \phi \wedge c)=a \cdot((\phi \wedge \neg \phi) \wedge(b+c))=\delta$ from the axioms of BPAps because, in the case of BPAps, $a \cdot(\phi \wedge b+\neg \phi \wedge c)$ is not capable of doing anything. We can only derive $a \cdot(\phi \wedge b+\neg \phi \wedge c)=a \cdot((\phi \wedge \neg \phi) \wedge(b+c))$ from the axioms of $\mathrm{BPA}_{\mathrm{ps}}^{\mathrm{ct}}$ because, in the case of $\mathrm{BPA}_{\mathrm{ps}}^{\mathrm{ct}}, a \cdot(\phi \wedge b+\neg \phi \wedge c)$ is capable of first performing $a$ and next either performing $b$ and after that terminating successfully or performing $c$ and after that terminating successfully - although the proposition that holds at the start of the process that remains after performing $a$ is the contradiction $\phi \wedge \neg \phi$.

Let $\phi$ be a closed term of sort $\mathbf{B}$ such that not $\vdash \phi \leftrightarrow \mathrm{F}$ and not $\vdash \neg \phi \leftrightarrow \mathrm{F}$. Then, because $\vdash \circ \phi \wedge \phi \wedge \neg \phi \leftrightarrow \mathrm{F}$, we have that $a \cdot(\circ \phi \wedge(\phi \wedge x+\neg \phi \wedge y))=$ $a \cdot(\mathrm{F} \wedge(x+y))=\delta$ is derivable from the axioms of $\mathrm{BPA}_{\mathrm{ps}}^{\mathrm{ct}}$. This shows that it can be enforced by means of a consistency proposition $(\circ \phi)$ that the alternative composition of two processes of which the propositions that hold at the start of them are contradictory leads to an inconsistency in $\mathrm{BPA}_{\mathrm{ps}}^{\mathrm{ct}}$.

Hereafter, we will write $[\phi]$ for the equivalence class of $\phi$ modulo $\Leftrightarrow$. That is, $[\phi]=\{\psi \mid \phi \Leftrightarrow \psi\}$. Hence, $[\phi]=\{\psi \mid \vdash \phi \leftrightarrow \psi\}$.

All processes that can be described by a closed term of $\mathrm{BPA}_{\mathrm{ps}}^{\mathrm{ct}}$, can be described by a basic term. The set $\mathcal{B}$ of basic terms is inductively defined by the following rules:

$-\perp \in \mathcal{B}$

- if $\phi \notin[\mathrm{F}]$, then $\phi \wedge \delta \in \mathcal{B}$;

- if $\phi \notin[\mathrm{F}]$ and $a \in \mathrm{A}$, then $\phi: \rightarrow a \in \mathcal{B}$;

- if $\phi \notin[\mathrm{F}], a \in \mathrm{A}$, and $p \in \mathcal{B}$, then $\phi: \rightarrow a \cdot p \in \mathcal{B}$;

- if $p, q \in \mathcal{B}$, then $p+q \in \mathcal{B}$. 
Each basic term can be written as $\perp$ or in the form

$$
\chi^{\wedge} \delta+\sum_{i \in\{1, \ldots, n\}} \phi_{i}: \rightarrow a_{i} \cdot p_{i}+\sum_{j \in\{1, \ldots, m\}} \psi_{j}: \rightarrow b_{j},
$$

where $n, m \in \mathbb{N}$, where $\chi \notin[\mathrm{F}]$, where $\phi_{i} \notin[\mathrm{F}], a_{i} \in \mathrm{A}$, and $p_{i} \in \mathcal{B}$ for all $i \in\{1, \ldots, n\}$, and where $\psi_{j} \notin[\mathrm{F}]$ and $b_{j} \in \mathrm{A}$ for all $j \in\{1, \ldots, m\}$. The subterm $\chi$ is called the root signal of the basic term and the subterms $\phi_{i}: \rightarrow a_{i} \cdot p_{i}$ and $\psi_{j}: \rightarrow b_{j}$ are called the summands of the basic term.

All closed $\mathrm{BPA}_{\mathrm{ps}}^{\mathrm{ct}}$ terms of sort $\mathbf{P}$ can be reduced to a basic term.

Proposition 2 (Elimination). For all closed $\mathrm{BPA}_{\mathrm{ps}}^{\mathrm{ct}}$ terms $p$ of sort $\mathbf{P}$, there exists a $q \in \mathcal{B}$ such that $p=q$ is derivable from the axioms of $\mathrm{BPA}_{\mathrm{ps}}^{\mathrm{ct}}$.

Proof. The proof is straightforward by induction on the structure of closed term $p$. If $p$ is of the form $\perp, a, p^{\prime}+p^{\prime \prime}$ or $\phi^{\wedge} p^{\prime}$, then it is trivial to show that there exists a $q \in \mathcal{B}$ such that $p=q$ is derivable from the axioms of $\mathrm{BPA}_{\mathrm{ps}}^{\mathrm{ct}}$. If $p$ is of the form $p^{\prime} \cdot p^{\prime \prime}$ or $\phi: \rightarrow p^{\prime}$, then it follows immediately from the induction hypothesis and the following claims:

- for all $p, p^{\prime} \in \mathcal{B}$, there exists a $p^{\prime \prime} \in \mathcal{B}$ such that $p \cdot p^{\prime}=p^{\prime \prime}$ is derivable from the axioms of $\mathrm{BPA}_{\mathrm{ps}}^{\mathrm{ct}}$;

- for all $\phi \notin[\mathrm{F}]$ and $p \in \mathcal{B}$, there exists a $p^{\prime} \in \mathcal{B}$ such that $\phi: \rightarrow p=p^{\prime}$ is derivable from the axioms of $\mathrm{BPA}_{\mathrm{ps}}^{\mathrm{ct}}$.

Both claims are easily proved by induction on the structure of basic term $p$.

\section{Semantics of $\mathrm{BPA}_{\mathrm{ps}}^{\mathrm{ct}}$}

In this section, we present a structural operational semantics of $\mathrm{BPA}_{\mathrm{ps}}^{\mathrm{ct}}$, define a notion of bisimulation equivalence based on this semantics, and show that the axioms of $\mathrm{BPA}_{\mathrm{ps}}^{\mathrm{ct}}$ are sound and complete with respect to this bisimulation equivalence.

We start with the presentation of the structural operational semantics of $\mathrm{BPA}_{\mathrm{ps}}^{\mathrm{ct}}$. The following transition relations on closed terms of sort $\mathbf{P}$ are used:

- for each $\ell \in C \times \mathrm{A}$, a binary action step relation $\stackrel{\ell}{\rightarrow}$;

- for each $\ell \in C \times \mathrm{A}$, a unary action termination relation $\stackrel{\ell}{\rightarrow} \sqrt{ }$;

- for each $\phi \in C$, a unary signal emission relation $\mathrm{s}^{\phi}$;

where $C$ is the set of all closed terms $\phi$ of sort $\mathbf{B}$ such that $\phi \notin[\mathrm{F}]$. We write $p \stackrel{\{\phi\} a}{\longrightarrow} q$ instead of $(p, q) \in \stackrel{(\phi, a)}{\longrightarrow}, p \stackrel{\{\phi\} a}{\longrightarrow} \sqrt{ }$ instead of $p \in \stackrel{(\phi, a)}{\longrightarrow} \sqrt{ }$, and $\mathbf{s}(p)=\phi$ instead of $p \in \mathbf{s}^{\phi}$. These relations can be explained as follows:

$-p \stackrel{\{\phi\} a}{\longrightarrow} \sqrt{ }: p$ is capable of performing action $a$ under condition $\phi$ and then terminating successfully; 
Table 4. Transition rules for $\mathrm{BPA}_{\mathrm{ps}}^{\mathrm{ct}}$

$$
\begin{aligned}
& \underset{a \stackrel{\{\mathrm{T}\} a}{\longrightarrow} \sqrt{ }}{\stackrel{\{\phi\} a}{a}} \\
& \frac{x \stackrel{\{\phi\} a}{\longrightarrow} \sqrt{ }, \mathrm{s}(x+y)=\psi}{x+y \stackrel{\{\phi\} a}{\longrightarrow} \sqrt{ }} \psi \notin[\mathrm{F}] \quad \frac{y \stackrel{\{\phi\} a}{\longrightarrow} \sqrt{ }, \mathrm{s}(x+y)=\psi}{x+y \stackrel{\{\phi\} a}{\longrightarrow} \sqrt{ }} \psi \notin[\mathrm{F}] \\
& \frac{x \stackrel{\{\phi\} a}{\longrightarrow} x^{\prime}, \mathrm{s}(x+y)=\psi}{x+y \stackrel{\{\phi\} a}{\longrightarrow} x^{\prime}} \psi \notin[\mathrm{F}] \quad \frac{y \stackrel{\{\phi\} a}{\longrightarrow} y^{\prime}, \mathrm{s}(x+y)=\psi}{x+y \stackrel{\{\phi\} a}{\longrightarrow} y^{\prime}} \psi \notin[\mathrm{F}] \\
& \frac{x \stackrel{\{\phi\} a}{\longrightarrow} \sqrt{ }, \mathrm{s}(y)=\psi}{x \cdot y \stackrel{\{\phi\} a}{\longrightarrow} y} \psi \notin[\mathrm{F}] \quad \frac{x \stackrel{\{\phi\} a}{\longrightarrow} x^{\prime}}{x \cdot y \stackrel{\{\phi\} a}{\longrightarrow} x^{\prime} \cdot y} \\
& \frac{x \stackrel{\{\phi\} a}{\longrightarrow} \sqrt{ }}{\psi: \rightarrow x \stackrel{\{\phi \wedge \psi\} a}{\longrightarrow} \sqrt{ }} \phi \wedge \psi \notin[\mathrm{F}] \quad \frac{x \stackrel{\{\phi\} a}{\longrightarrow} x^{\prime}}{\psi: \rightarrow x \stackrel{\{\phi \wedge \psi\} a}{\longrightarrow} x^{\prime}} \phi \wedge \psi \notin[\mathrm{F}] \\
& \frac{x \stackrel{\{\phi\} a}{\longrightarrow} \sqrt{ }, \mathrm{s}(\psi \wedge x)=\chi}{\psi \wedge x \stackrel{\{\phi\} a}{\longrightarrow} \sqrt{ }} \chi \notin[\mathrm{F}] \quad \frac{x \stackrel{\{\phi\} a}{\longrightarrow} x^{\prime}, \mathrm{s}(\psi \wedge x)=\chi}{\psi \wedge x \stackrel{\{\phi\} a}{\longrightarrow} x^{\prime}} \chi \notin[\mathrm{F}] \\
& \overline{\mathrm{s}(\perp)=\mathrm{F}} \quad \overline{\mathrm{s}(a)=\mathrm{T}} \\
& \begin{array}{llll}
\frac{\mathbf{s}(x)=\phi, \mathbf{s}(y)=\psi}{\mathbf{s}(x+y)=\phi \wedge \psi} & \frac{\mathbf{s}(x)=\phi}{\mathbf{s}(x \cdot y)=\phi} & \frac{\mathbf{s}(x)=\phi}{\mathbf{s}(\psi: \rightarrow y)=\psi \supset \phi} & \frac{\mathbf{s}(x)=\phi}{\mathbf{s}(\psi \wedge y)=\psi \wedge \phi} \\
\hline
\end{array}
\end{aligned}
$$

$-p \stackrel{\{\phi\}^{a}}{\longrightarrow} q: p$ is capable of performing action $a$ under condition $\phi$ and then proceeding as $q$;

- $\mathrm{s}(p)=\phi$ : the proposition that holds at the start of $p$ is $\phi$.

The structural operational semantics of $\mathrm{BPA}_{\mathrm{ps}}^{\mathrm{ct}}$ is described by the transition rules given in Table 4. In this table, $a$ stands for an arbitrary constant from $\mathrm{A} \cup\{\delta\}$ and $\phi, \psi$, and $\chi$ stand for arbitrary closed terms of sort $\mathbf{B}$.

A bisimulation is a binary relation $R$ on closed $\mathrm{BPA}_{\mathrm{ps}}^{\mathrm{ct}}$ terms of sort $\mathbf{P}$ such that, for all closed $\mathrm{BPA}_{\mathrm{ps}}^{\mathrm{ct}}$ terms $p, q$ of sort $\mathbf{P}$ with $(p, q) \in R$, the following conditions hold:

- if $p \stackrel{\{\phi\} a}{\longrightarrow} p^{\prime}$, then, for all valuations $\nu$ with $\nu(\mathrm{s}(p)) \neq \mathrm{f}$ and $\nu(\phi) \neq \mathrm{f}$, there exists a closed term $\psi$ of sort $\mathbf{B}$ and a closed term $q^{\prime}$ of sort $\mathbf{P}$ such that $\nu(\phi)=\nu(\psi), q \stackrel{\{\psi\} a}{\longrightarrow} q^{\prime}$, and $\left(p^{\prime}, q^{\prime}\right) \in R ;$

- if $q \stackrel{\{\psi\} a}{\longrightarrow} q^{\prime}$, then, for all valuations $\nu$ with $\nu(\mathrm{s}(q)) \neq \mathrm{f}$ and $\nu(\psi) \neq \mathrm{f}$, there exists a closed term $\phi$ of sort $\mathbf{B}$ and a closed term $p^{\prime}$ of sort $\mathbf{P}$ such that $\nu(\psi)=\nu(\phi), p \stackrel{\{\phi\} a}{\longrightarrow} p^{\prime}$, and $\left(p^{\prime}, q^{\prime}\right) \in R ;$

- if $p \stackrel{\{\phi\} a}{\longrightarrow} \sqrt{ }$, then, for all valuations $\nu$ with $\nu(\mathrm{s}(p)) \neq \mathrm{f}$ and $\nu(\phi) \neq \mathrm{f}$, there exists a closed term $\psi$ of sort $\mathbf{B}$ such that $\nu(\phi)=\nu(\psi)$ and $q \stackrel{\{\psi\} a}{\longrightarrow} \sqrt{ }$;

- if $q \stackrel{\{\psi\} a}{\longrightarrow} \sqrt{ }$, then, for all valuations $\nu$ with $\nu(\mathrm{s}(q)) \neq \mathrm{f}$ and $\nu(\psi) \neq \mathrm{f}$, there exists a closed term $\phi$ of sort $\mathbf{B}$ such that $\nu(\psi)=\nu(\phi)$ and $p \stackrel{\{\phi\} a}{\longrightarrow} \sqrt{ }$; 
- if $\mathbf{s}(p)=\phi$, then there exists a closed term $\psi$ of sort $\mathbf{B}$ such that $\mathbf{s}(q)=\psi$ and $\phi \Leftrightarrow \psi$;

- if $\mathrm{s}(q)=\psi$, then there exists a closed term $\phi$ of sort $\mathbf{B}$ such that $\mathrm{s}(p)=\phi$ and $\psi \Leftrightarrow \phi$.

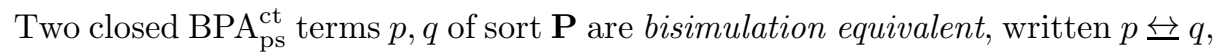
if there exists a bisimulation $R$ such that $(p, q) \in R$. Let $R$ be a bisimulation

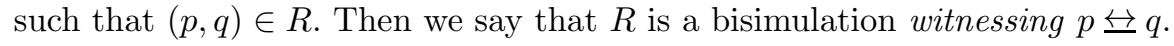

Henceforth, we will loosely say that a relation contains all closed substitution instances of an equation if it contains all pairs $\left(t, t^{\prime}\right)$ such that $t=t^{\prime}$ is a closed substitution instance of the equation.

Because a transition on one side may be simulated by a set of transitions on the other side, a bisimulation as defined above is called a splitting bisimulation in [15].

Bisimulation equivalence is a congruence with respect to the operators of $\mathrm{BPA}_{\mathrm{ps}}^{\mathrm{ct}}$.

Proposition 3 (Congruence). For all closed $\mathrm{BPA}_{\mathrm{ps}}^{\mathrm{ct}}$ terms $p, q, p^{\prime}, q^{\prime}$ of sort

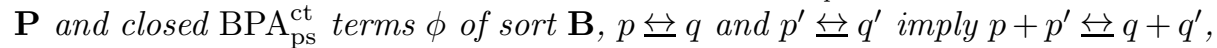

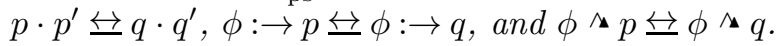

Proof. We can reformulate the transition rules such that:

- bisimulation equivalence based on the reformulated transition rules according to the standard definition of bisimulation equivalence coincides with bisimulation equivalence based on the original transition rules according to the definition of bisimulation equivalence given above;

- the reformulated transition rules make up a complete transition system specification in panth format.

The reformulation goes like the one for the transition rules for BPAps outlined in 8 . The proposition follows now immediately from the well-known result that bisimulation equivalence according to the standard definition of bisimulation equivalence is a congruence if the transition rules concerned make up a complete transition system specification in panth format (see e.g. [21]).

The underlying idea of the reformulation referred to above is that we replace each transition $p \stackrel{\{\phi\} a}{\longrightarrow} p^{\prime}$ by a transition $p \stackrel{\{\nu\} a}{\longrightarrow} p^{\prime}$ for each valuation $\nu$ such that $\nu(\phi) \neq \mathrm{f}$, and likewise $p \stackrel{\{\phi\} a}{\longrightarrow} \sqrt{ }$ and $\mathrm{s}(p)=\phi$. Thus, in a bisimulation, a transition on one side must be simulated by a single transition on the other side. We did not present the reformulated structural operational semantics in this paper because it is, in our opinion, intuitively less appealing.

$\mathrm{BPA}_{\mathrm{ps}}^{\mathrm{ct}}$ is sound with respect to $\leftrightarrows$ for equations between closed terms.

Theorem 1 (Soundness). For all closed $\mathrm{BPA}_{\mathrm{ps}}^{\mathrm{ct}}$ terms $p, q$ of sort $\mathbf{P}, p=q$ is

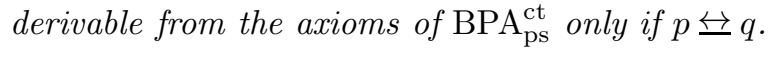


Proof. Because of Proposition 3 it is sufficient to prove the theorem for all closed substitution instances of each axiom of $\mathrm{BPA}_{\mathrm{ps}}^{\mathrm{ct}}$.

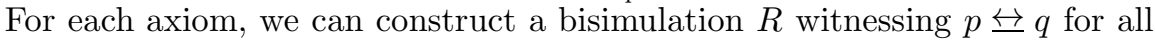
closed substitution instances $p=q$ of the axiom as follows:

- in the case of A1-A4 and A6, we take the relation $R$ that consists of all closed substitution instances of the axiom concerned and the equation $x=x$;

- in the case of A5, we take the relation $R$ that consists of all closed substitution instances of A5, SE5, and the equation $x=x$;

- in the case of A7, NE1-NE3, GC2-GC3, and SE2-SE3, we take the relation $R$ that consists of all closed substitution instances of the axiom concerned;

- in the case of GC1, GC4-GC7, SE1, and SE4-SE8, we take the relation $R$ that consists of all closed substitution instances of the axiom concerned and the equation $x=x$.

The laws from property (8) of $\mathrm{LP}^{\supset, \mathrm{F}}$ mentioned in Section 2 are needed to check that these relations are witnessing ones.

The proof of Theorem 1 goes along the same line as the soundness proof for BPAps outlined in 8 . The laws from property (8) of LP ${ }^{\supset}, \mathrm{F}$ mentioned in Section 2 are laws that $\mathrm{LP}^{\supset}, \mathrm{F}$ has in common with classical propositional logic. They are needed in the soundness proof for BPAps as well, but their use is left implicit in the proof outline given in 8 .

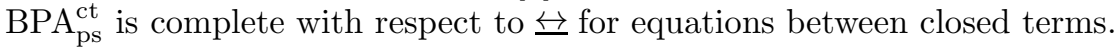

Theorem 2 (Completeness). For all closed $\mathrm{BPA}_{\mathrm{ps}}^{\mathrm{ct}}$ terms $p, q$ of sort $\mathbf{P}, p=q$

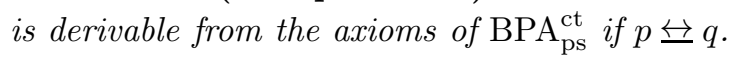

Proof. By Proposition 2 and Theorem 1, it is sufficient to prove the theorem for basic terms $p$ and $q$.

For $p, p^{\prime} \in \mathcal{B}, p^{\prime}$ is called a basic subterm of $p$ if $p^{\prime} \equiv p$ or there exists an $a \in \mathrm{A}$ such that $a \cdot p^{\prime}$ is a subterm of $p$.

We introduce a reduction relation $\gg$ on $\mathcal{B}$. The one-step reduction relation $\longmapsto$ on $\mathcal{B}$ is inductively defined as follows:

- if $p^{\prime}$ is a basic subterm of $p$ and $q^{\prime}$ occurs twice as summand in $p^{\prime}$, then $p \longmapsto r$ where $r$ is $p$ with one occurrence of $q^{\prime}$ removed;

- if $p^{\prime}$ is a basic subterm of $p$ and both $\phi: \rightarrow a \cdot q^{\prime}$ and $\psi: \rightarrow a \cdot q^{\prime}$ occur as summand in $p^{\prime}$, then $p \longmapsto r$ where $r$ is $p$ with the occurrence of $\phi: \rightarrow a \cdot q^{\prime}$ replaced by $\phi \vee \psi: \rightarrow a \cdot q^{\prime}$ and the occurrence of $\psi: \rightarrow a \cdot q^{\prime}$ removed;

- if $p^{\prime}$ is a basic subterm of $p$ and both $\phi: \rightarrow a$ and $\psi: \rightarrow a$ occur as summand in $p^{\prime}$, then $p \longmapsto r$ where $r$ is $p$ with the occurrence of $\phi: \rightarrow a$ replaced by $\phi \vee \psi: \rightarrow a$ and the occurrence of $\psi: \rightarrow a$ removed.

The one-step reductions correspond to sharing of double states and joining of transitions as in [16. The reduction relation $\succcurlyeq$ is the reflexive and transitive closure of $\hookrightarrow$, and the conversion relation $x \rightarrow$ is the reflexive and transitive closure of $\iota \cup \longmapsto^{-1}$.

The following are important properties of $\longrightarrow$ : 
(1) $\rightsquigarrow$ is strongly normalizing;

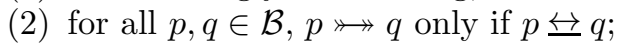

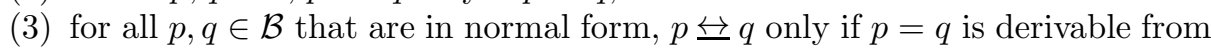
axioms $\mathrm{A} 1$ and $\mathrm{A} 2$;

(4) for all $p, q \in \mathcal{B}, p \longmapsto q$ only if $p=q$ is derivable from the axioms of $\mathrm{BPA}_{\mathrm{ps}}^{\mathrm{ct}}$.

Verifying properties (1), (2), and (4) is trivial. Property (3) can be verified by proving it, simultaneously with the property

for all $p \in \mathcal{B}$ that are in normal form, any bisimulation between $p$ and itself is the identity relation,

by induction on the number of occurrences of a constant from $\mathrm{A}$ in $p$ and $q$. The proof is similar to the proof of Theorem 2.12 from [13, but easier.

From properties (1), (2) and (3), it follows immediately that, for all $p, q \in \mathcal{B}$,

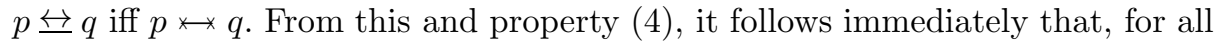

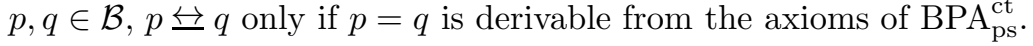

\section{Contradiction-Tolerant ACP with Propositional Signals}

In this section, we present the contradiction-tolerant version of ACPps. This version, which is called $\mathrm{ACP}_{\mathrm{ps}}^{\mathrm{ct}}$, is an extension of $\mathrm{BPA}_{\mathrm{ps}}^{\mathrm{ct}}$ that supports parallelism and communication.

In $\mathrm{ACP}_{\mathrm{ps}}^{\mathrm{ct}}$, just as in $\mathrm{BPA}_{\mathrm{ps}}^{\mathrm{ct}}$, it is assumed that a fixed but arbitrary finite set $\mathrm{A}$ of actions, with $\delta \notin \mathrm{A}$, and a fixed but arbitrary finite set $\mathrm{B}_{\text {at }}$ of atomic propositions have been given. In $\mathrm{ACP}_{\mathrm{ps}}^{\mathrm{ct}}$, it is further assumed that a fixed but arbitrary commutative and associative communication function $\mid: \mathrm{A}_{\delta} \times \mathrm{A}_{\delta} \rightarrow \mathrm{A}_{\delta}$, such that $\delta \mid a=\delta$ for all $a \in \mathrm{A}_{\delta}$, has been given. The function $\mid$ is regarded to give the result of synchronously performing any two actions for which this is possible, and to be $\delta$ otherwise.

The algebraic theory $\mathrm{ACP}_{\mathrm{ps}}^{\mathrm{ct}}$ has the sorts, constants and operators of $\mathrm{BPA}_{\mathrm{ps}}^{\mathrm{ct}}$ and in addition the following operators:

- the binary parallel composition operator $\|: \mathbf{P} \times \mathbf{P} \rightarrow \mathbf{P}$;

- the binary left merge operator $\llbracket: \mathbf{P} \times \mathbf{P} \rightarrow \mathbf{P}$;

- the binary communication merge operator $\mid: \mathbf{P} \times \mathbf{P} \rightarrow \mathbf{P}$;

- for each $H \subseteq \mathrm{A}$, the unary encapsulation operator $\partial_{H}: \mathbf{P} \rightarrow \mathbf{P}$.

We use infix notation for the additional binary operators as well.

The constants and operators of $\mathrm{ACP}_{\mathrm{ps}}^{\mathrm{ct}}$ to build terms of sort $\mathbf{P}$ are the constants and operators of ACP and additionally the guarded command operator and the signal emission operator.

Let $p$ and $q$ be closed terms of sort $\mathbf{P}$. Intuitively, the additional operators can be explained as follows:

- $p \| q$ behaves as the process that proceeds with $p$ and $q$ in parallel, the proposition that holds at the start of $p \| q$ is the conjunction of the propositions that hold at the start of $p$ and $q$; 
Table 5. Additional axioms for $\mathrm{ACP}_{\mathrm{ps}}^{\mathrm{ct}}$

\begin{tabular}{|c|c|c|c|}
\hline$x \| y=x \llbracket y+y \llbracket x+x \mid y$ & CM1 & $a|b=b| a$ & $\mathrm{C} 1$ \\
\hline$a \llbracket x=a \cdot x+\partial_{\mathrm{A}}(x)$ & $\mathrm{CM} 2 \mathrm{~S}$ & $(a \mid b)|c=a|(b \mid c)$ & $\mathrm{C} 2$ \\
\hline$a \cdot x \llbracket y=a \cdot(x \| y)+\partial_{\mathrm{A}}(y)$ & CM3S & $\delta \mid a=\delta$ & $\mathrm{C} 3$ \\
\hline$(x+y) \llbracket z=x \llbracket z+y \llbracket z$ & $\mathrm{CM} 4$ & & \\
\hline$a \cdot x \mid b=(a \mid b) \cdot x$ & CM5 & & \\
\hline$a \mid b \cdot x=(a \mid b) \cdot x$ & CM6 & $\partial_{H}(a)=a$ & D1 \\
\hline$a \cdot x \mid b \cdot y=(a \mid b) \cdot(x \| y)$ & CM7 & $\partial_{H}(a)=\delta$ & D2 \\
\hline$(x+y)|z=x| z+y \mid z$ & CM8 & $\partial_{H}(x+y)=\partial_{H}(x)+\partial_{H}(y)$ & D3 \\
\hline$x|(y+z)=x| y+x \mid z$ & CM9 & $\partial_{H}(x \cdot y)=\partial_{H}(x) \cdot \partial_{H}(y)$ & $\mathrm{D} 4$ \\
\hline$(\phi: \rightarrow x) \llbracket y=\phi: \rightarrow(x \llbracket y)+\partial_{\mathrm{A}}(y)$ & GC8S & $(\phi \wedge x) \llbracket y=\phi \wedge(x \Downarrow y)$ & SE9 \\
\hline$(\phi: \rightarrow x) \mid y=\phi: \rightarrow(x \mid y)+\partial_{\mathrm{A}}(y)$ & GC9S & $(\phi \wedge x) \mid y=\phi \wedge(x \mid y)$ & SE10 \\
\hline$x \mid(\phi: \rightarrow y)=\phi: \rightarrow(x \mid y)+\partial_{\mathrm{A}}(x)$ & GC10S & $x \mid(\phi \wedge y)=\phi \wedge(x \mid y)$ & SE11 \\
\hline$\partial_{H}(\phi: \rightarrow x)=\phi: \rightarrow \partial_{H}(x)$ & GC11 & $\partial_{H}(\phi \wedge x)=\phi \wedge \partial_{H}(x)$ & SE12 \\
\hline
\end{tabular}

- $p \llbracket q$ behaves the same as $p \| q$, except that it starts with performing an action of $p$, the proposition that holds at the start of $p \llbracket q$ is the conjunction of the propositions that hold at the start of $p$ and $q$;

- $p \mid q$ behaves the same as $p \| q$, except that it starts with performing an action of $p$ and an action of $q$ synchronously, the proposition that holds at the start of $p \mid q$ is the conjunction of the propositions that hold at the start of $p$ and $q$;

- $\partial_{H}(p)$ behaves the same as $p$, except that the actions in $H$ are blocked, the proposition that holds at the start of $\partial_{H}(p)$ is the proposition that holds at the start of $p$.

The axioms of $\mathrm{ACP}_{\mathrm{ps}}^{\mathrm{ct}}$ are the axioms of $\mathrm{BPA}_{\mathrm{ps}}^{\mathrm{ct}}$ and the additional axioms given in Table 5. In this table, $a, b, c$ stand for arbitrary constants from $\mathrm{A} \cup\{\delta\}$ and $\phi$ stands for an arbitrary closed term of sort B. A1-A7, CM1-CM9 with CM1S and CM2S replaced by $a \llbracket x=a \cdot x$ and $a \cdot x \llbracket y=a \cdot(x \| y), \mathrm{C} 1-\mathrm{C} 3$, and D1-D4 are the axioms of ACP (see e.g. 11]). GC11 and SE9-SE12 have been taken from [8] and GC9S and GC10S have been taken from [8] with subterms of the form $\mathrm{s}(x) \wedge \delta$ replaced by $\partial_{\mathrm{A}}(x)$. CM2S, CM3S and GC8S differ really from the corresponding axioms in $[8$ due to the choice of having as the proposition that holds at the start of the left merge of two processes, as in the case of the communication merge, always the conjunction of the propositions that hold at the start of the two processes.

The following equations are among the equations derivable from the axioms of $\mathrm{ACP}_{\mathrm{ps}}^{\mathrm{ct}}$ :

$$
\begin{gathered}
(\phi \wedge x) \|(\psi \wedge y)=(\phi \wedge \psi) \wedge(x \| y), \\
x\|\perp=\perp, \quad \perp\| x=\perp .
\end{gathered}
$$


Let $\phi$ be a closed term of sort $\mathbf{B}$ such that not $\vdash \phi \leftrightarrow \mathrm{F}$ and not $\vdash \neg \phi \leftrightarrow \mathrm{F}$. Then, because not $\vdash \phi \wedge \neg \phi \leftrightarrow \mathrm{F}$, we have that $a \cdot\left(\phi^{\wedge} x \| \neg \phi^{\wedge} y\right)=a \cdot(\mathrm{F} \wedge(x \| y))=$ $\delta$, which is derivable from the axioms of ACPps, is not derivable from the axioms of $\mathrm{ACP}_{\mathrm{ps}}^{\mathrm{ct}}$. This shows the main difference between $\mathrm{ACP}_{\mathrm{ps}}^{\mathrm{ct}}$ and ACPps: the parallel composition of two processes of which the propositions that hold at the start of them are contradictory does not lead to an inconsistency in $\mathrm{ACP}_{\mathrm{ps}}^{\mathrm{ct}}$, whereas it does lead to an inconsistency in ACPps. This is why $\mathrm{ACP}_{\mathrm{ps}}^{\mathrm{ct}}$ is called the contradiction-tolerant version of ACPps.

Let $\phi$ be a closed term of sort B such that not $\vdash \phi \leftrightarrow \mathrm{F}$ and not $\vdash \neg \phi \leftrightarrow \mathrm{F}$. Assume that $b \mid c=d$. Then we can derive $a \cdot(\phi \wedge b \| \neg \phi \wedge c)=a \cdot((\phi \wedge \neg \phi) \wedge(b \| c))=$ $a \cdot((\phi \wedge \neg \phi) \wedge(b \cdot c+c \cdot b+d))=\delta$ from the axioms of BPAps because, in the case of BPAps, $a \cdot(\phi \wedge b \| \neg \phi \wedge c)$ is not capable of doing anything. We can only derive $a \cdot(\phi \wedge b \| \neg \phi \wedge c)=a \cdot((\phi \wedge \neg \phi) \wedge(b \| c))=a \cdot((\phi \wedge \neg \phi) \wedge(b \cdot c+c \cdot b+d))$ from the axioms of $\mathrm{BPA}_{\mathrm{ps}}^{\mathrm{ct}}$ because, in the case of $\mathrm{BPA}_{\mathrm{ps}}^{\mathrm{ct}}, a \cdot(\phi \wedge b \| \neg \phi \wedge c)$ is capable of first performing $a$ and next either performing $b$ and $c$ in either order and after that terminating successfully or performing $d$ and after that terminating successfully - although the proposition that holds at the start of the process that remains after performing $a$ is the contradiction $\phi \wedge \neg \phi$.

Let $\phi$ be a closed term of sort $\mathbf{B}$ such that not $\vdash \phi \leftrightarrow \mathrm{F}$ and not $\vdash \neg \phi \leftrightarrow \mathrm{F}$. Then, because $\vdash \circ \phi \wedge \phi \wedge \neg \phi \leftrightarrow \mathrm{F}$, we have that $a \cdot(\circ \phi \wedge(\phi \wedge x \| \neg \phi \wedge y))=$ $a \cdot(\mathrm{F} \wedge(x \| y))=\delta$ is derivable from the axioms of $\mathrm{ACP}_{\mathrm{ps}}^{\mathrm{ct}}$. This shows that it can be enforced by means of a consistency proposition $(\circ \phi)$ that the parallel composition of two processes of which the propositions that hold at the start of them are contradictory leads to an inconsistency in $\mathrm{ACP}_{\mathrm{ps}}^{\mathrm{ct}}$.

All closed $\mathrm{ACP}_{\mathrm{ps}}^{\mathrm{ct}}$ terms of sort $\mathbf{P}$ can be reduced to a basic term.

Proposition 4 (Elimination). For all closed $\mathrm{ACP}_{\mathrm{ps}}^{\mathrm{ct}}$ terms $p$ of sort $\mathbf{P}$, there exists a $q \in \mathcal{B}$ such that $p=q$ is derivable from the axioms of $\mathrm{ACP}_{\mathrm{ps}}^{\mathrm{ct}}$.

Proof. The proof is straightforward by induction on the structure of closed term $p$. If $p$ is of the form $\perp, a, p^{\prime}+p^{\prime \prime}, p^{\prime} \cdot p^{\prime \prime}, \phi: \rightarrow p^{\prime}$ or $\phi p^{\prime}$, then it follows immediately from the induction hypothesis and Proposition 2 that there exists a $q \in \mathcal{B}$ such that $p=q$ is derivable from the axioms of $\mathrm{ACP}_{\mathrm{ps}}^{\mathrm{ct}}$. If $p$ is of the form $p^{\prime} \| p^{\prime \prime}, p^{\prime} \llbracket p^{\prime \prime}, p^{\prime} \mid p^{\prime \prime}$ or $\partial_{H}\left(p^{\prime}\right)$, then it follows immediately from the induction hypothesis and claims similar to the ones from the proof of Proposition 2 The

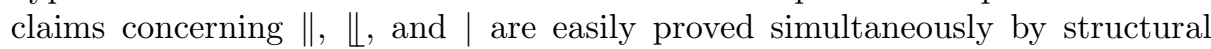
induction. The claim concerning $\partial_{H}$ is easily proved by structural induction.

\section{Semantics of $\mathrm{ACP}_{\mathrm{ps}}^{\mathrm{ct}}$}

In this section, we present a structural operational semantics of $\mathrm{ACP}_{\mathrm{ps}}^{\mathrm{ct}}$ and show that the axioms of $\mathrm{ACP}_{\mathrm{ps}}^{\mathrm{ct}}$ are sound and complete with respect to this bisimulation equivalence.

We start with the presentation of the structural operational semantics of $\mathrm{ACP}_{\mathrm{ps}}^{\mathrm{ct}}$. The structural operational semantics of $\mathrm{ACP}_{\mathrm{ps}}^{\mathrm{ct}}$ is described by the transition rules for $\mathrm{BPA}_{\mathrm{ps}}^{\mathrm{ct}}$ and the additional transition rules given in Table 6] In 
Table 6. Additional transition rules for $\mathrm{ACP}_{\mathrm{ps}}^{\mathrm{ct}}$

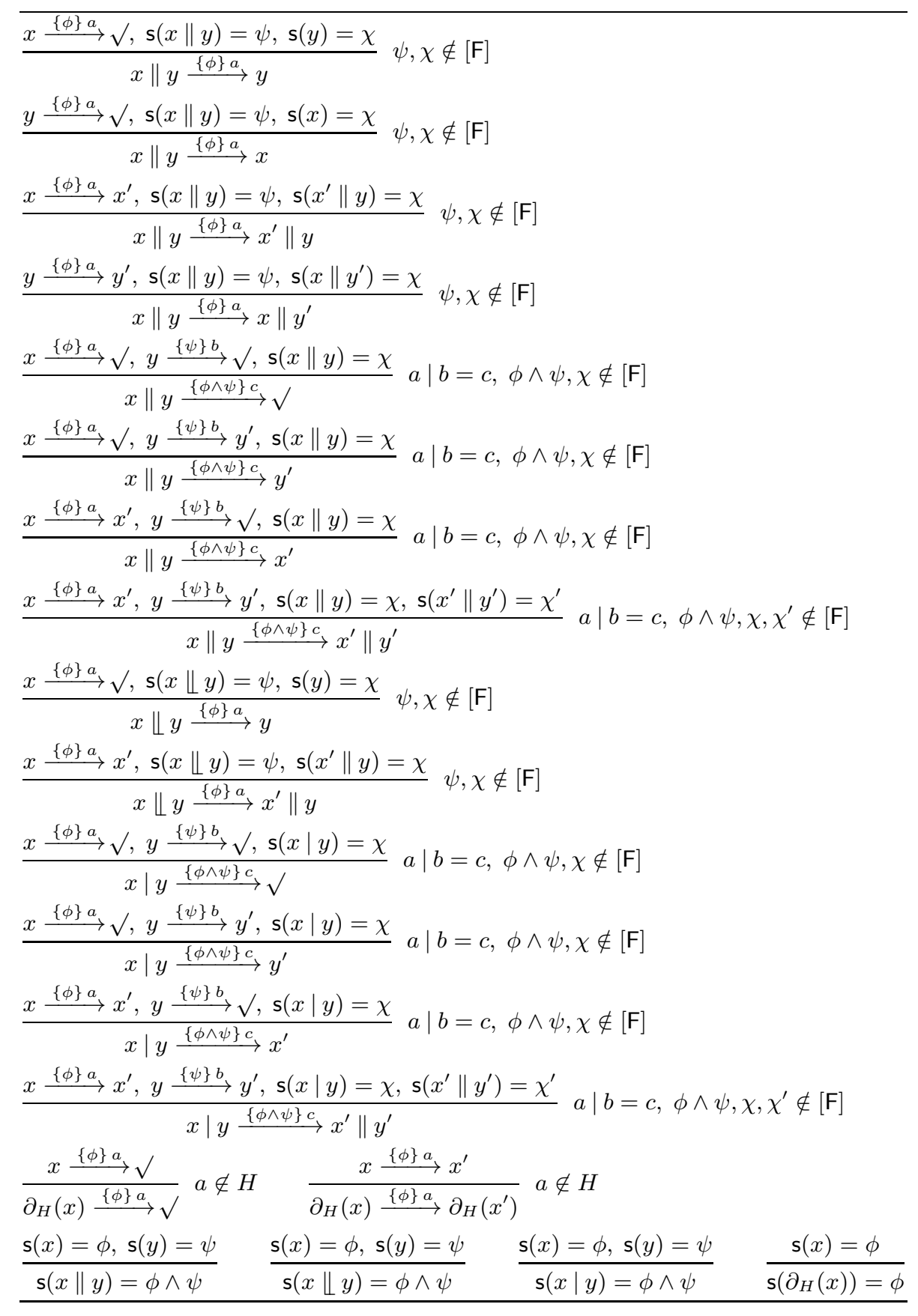


these tables, $a, b$, and $c$ stand for arbitrary constants from $\mathrm{A} \cup\{\delta\}$ and $\phi, \psi, \chi$, and $\chi^{\prime}$ stand for arbitrary closed terms of sort $\mathbf{B}$.

In Sections 3 and 5 , we have touched upon the main difference between $\mathrm{ACP}_{\mathrm{ps}}^{\mathrm{ct}}$ and ACPps: the alternative and parallel composition of two processes of which the propositions that hold at the start of them are contradictory does not lead to an inconsistency in $\mathrm{ACP}_{\mathrm{ps}}^{\mathrm{ct}}$, whereas it does lead to an inconsistency in ACPps. However, the transition rules for $\mathrm{ACP}_{\mathrm{ps}}^{\mathrm{ct}}$ and $\mathrm{ACP} p s$ seem to be the same. The difference is fully accounted for by the fact that $[F]$, the equivalence class of $F$ modulo logical equivalence, contains in the case of $\mathrm{LP}^{\supset, \mathrm{F}}$ only propositions of the form $\phi \wedge \neg \phi$ with $\phi$ such that either $\phi \Leftrightarrow \mathrm{F}$ or $\neg \phi \Leftrightarrow \mathrm{F}$, whereas it contains in the case of classical propositional logic all propositions of the form $\phi \wedge \neg \phi$.

By this fact, in the case of $\mathrm{ACP}_{\mathrm{ps}}^{\mathrm{ct}}, a \cdot(\phi \wedge b \| \neg \phi \wedge c)$ from the example preceding Proposition 4 is capable of first performing $a$ and next either performing $b$ and $c$ in either order and after that terminating successfully or performing $d$ and after that terminating successfully - although the proposition that holds at the start of the process that remains after performing $a$ is the contradiction $\phi \wedge \neg \phi-$ and, in the case of ACPps, it is not capable of doing anything.

Bisimulation equivalence is a congruence with respect to the operators of $\mathrm{ACP}_{\mathrm{ps}}^{\mathrm{ct}}$.

Proposition 5 (Congruence). For all closed $\mathrm{ACP}_{\mathrm{ps}}^{\mathrm{ct}}$ terms $p, q, p^{\prime}, q^{\prime}$ of sort $\mathbf{P}$ and closed $\mathrm{ACP}_{\mathrm{ps}}^{\mathrm{ct}}$ terms $\phi$ of sort $\mathbf{B}, p \leftrightarrows q$ and $p^{\prime} \leftrightarrows q^{\prime}$ imply $p+p^{\prime} \leftrightarrows q+q^{\prime}$,

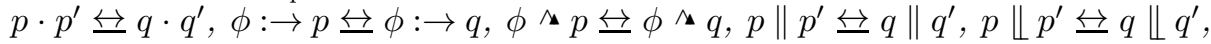

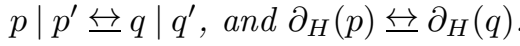

Proof. The proof goes along the same line as the proof of Proposition 3

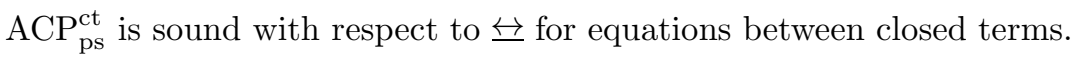

Theorem 3 (Soundness). For all closed $\mathrm{ACP}_{\mathrm{ps}}^{\mathrm{ct}}$ terms $p, q$ of sort $\mathbf{P}, p=q$ is

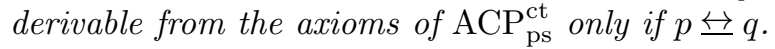

Proof. Because of Proposition 5 it is sufficient to prove the theorem for all closed substitution instances of each axiom of $\mathrm{ACP}_{\mathrm{ps}}^{\mathrm{ct}}$.

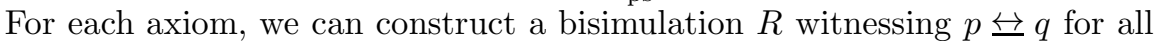
closed substitution instances $p=q$ of the axiom as follows:

- in the case of the axioms of $\mathrm{BPA}_{\mathrm{ps}}^{\mathrm{ct}}$, we take the same relation as in the proof of Theorem 1

- in the case of CM1, we take the relation $R$ that consists of all closed substitution instances of CM1, the equation $x\|y=y\| x$, and the equation $x=x$

- in the case of CM2S-CM9, we take the relation $R$ that consists of all closed substitution instances of the axiom concerned and the equation $x=x$;

- in the case of C1-C3 and D1-D2, we take the relation $R$ that consists of all closed substitution instances of the axiom concerned; 
- in the case of D3-D4, GC8S-GC11, and SE9-SE12, we take the relation $R$ that consists of all closed substitution instances of the axiom concerned and the equation $x=x$.

The laws from property (8) of $\mathrm{LP}^{\mathrm{D}, \mathrm{F}}$ mentioned in Section 2 are needed to check that these relations are witnessing ones.

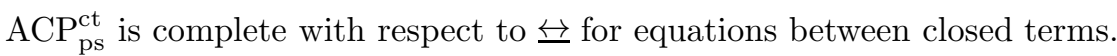

Theorem 4 (Completeness). For all closed $\mathrm{ACP}_{\mathrm{ps}}^{\mathrm{ct}}$ terms $p, q$ of sort $\mathbf{P}, p=q$

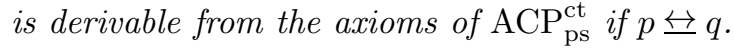

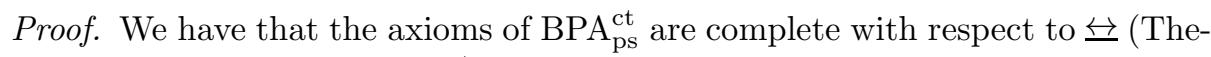

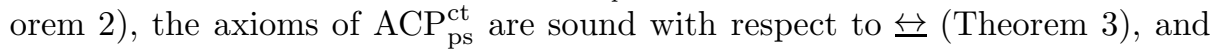
for each closed $\mathrm{ACP}_{\mathrm{ps}}^{\mathrm{ct}}$ term $p$ of sort $\mathbf{P}$, there exists a closed $\mathrm{BPA}_{\mathrm{ps}}^{\mathrm{ct}}$ term $q$ such that $p=q$ is derivable from the axioms of $\mathrm{ACP}_{\mathrm{ps}}^{\mathrm{ct}}$ (Proposition 4). By Theorem 3.14 from [31], the result immediately follows from this and the claim that the set of transition rules for $\mathrm{ACP}_{\mathrm{ps}}^{\mathrm{ct}}$ is an operational conservative extension of the set of transition rules for $\mathrm{BPA}_{\mathrm{ps}}^{\mathrm{ct}}$.

This claim can easily be proved if we reformulate the transition rules for $\mathrm{ACP}_{\mathrm{ps}}^{\mathrm{ct}}$ in the same way as the transition rules for $\mathrm{BPA}_{\mathrm{ps}}^{\mathrm{ct}}$ have been reformulated to prove Proposition 3. The operational conservativity can then easily be proved by verifying that the reformulated transition rules for $\mathrm{ACP}_{\mathrm{ps}}^{\mathrm{ct}}$ makes up a complete transition system specification, the reformulated transition rules for $\mathrm{BPA}_{\mathrm{ps}}^{\mathrm{ct}}$ - which are included in the reformulated transition rules for $\mathrm{ACP}_{\mathrm{ps}}^{\mathrm{ct}}-$ are source-dependent, and the additional transition rules have fresh sources (see e.g. [22]).

\section{State Operators}

In this section, we extend $\mathrm{ACP}_{\mathrm{ps}}^{\mathrm{ct}}$ with state operators. The resulting theory is called $\mathrm{ACP}_{\mathrm{ps}}^{\mathrm{ct}}+\mathrm{SO}$. The state operators introduced here generalize the state operators added to ACP in [6].

The state operators from [ 6] were introduced to make it easy to represent the execution of a process in a state. The basic idea was that the execution of an action in a state has effect on the state, i.e. it causes a change of state. Moreover, there is an action left when an action is executed in a state. The main difference between the original state operators and the state operators introduced here is that, in the case of the latter, the state in which a process is executed determines the proposition that holds at its start. Thus, one application of a state operator may replace many applications of the signal emission operator.

It is assumed that a fixed but arbitrary set $S$ of states has been given, together with functions act: A $\times S \rightarrow \mathrm{A}_{\delta}$, eff : A $\times S \rightarrow S$, and sig: $S \rightarrow B$, where $B$ is the set of all closed terms $\phi$ of sort $\mathbf{B}$.

For each $s \in S$, we add a unary state operator $\lambda_{s}: \mathbf{P} \rightarrow \mathbf{P}$ to the operators of $\mathrm{ACP}_{\mathrm{ps}}^{\mathrm{ct}}$. 
Table 7. Axioms for state operators

\begin{tabular}{ll}
\hline$\lambda_{s}(a)=\operatorname{sig}(s) \wedge \operatorname{act}(a, s)$ & SO1 \\
$\lambda_{s}(a \cdot x)=\operatorname{sig}(s) \wedge \operatorname{act}(a, s) \cdot \lambda_{\text {eff }(a, s)}(x)$ & SO2 \\
$\lambda_{s}(x+y)=\lambda_{s}(x)+\lambda_{s}(y)$ & SO3 \\
$\lambda_{s}(\phi: \rightarrow x)=\operatorname{sig}(s) \wedge\left(\phi: \rightarrow \lambda_{s}(x)\right)$ & SO4 \\
$\lambda_{s}(\phi \wedge x)=\phi \wedge \lambda_{s}(x)$ & SO5 \\
\hline
\end{tabular}

Table 8. Transition rules for state operators

$$
\begin{aligned}
& \overline{\frac{x \stackrel{\{\phi\} a}{\longrightarrow} \sqrt{ }, \mathrm{s}\left(\lambda_{s}(x)\right)=\psi}{\lambda_{s}(x) \stackrel{\{\phi\} \operatorname{act}(a, s)}{\longrightarrow}} \sqrt{ }} \operatorname{act}(a, s) \neq \delta, \psi \notin[\mathrm{F}] \\
& \frac{x \stackrel{\{\phi\} a}{\longrightarrow} x^{\prime}, \mathrm{s}\left(\lambda_{s}(x)\right)=\psi, \mathrm{s}\left(\lambda_{\operatorname{eff}(a, s)}\left(x^{\prime}\right)\right)=\chi}{\lambda_{s}(x) \stackrel{\{\phi\} \operatorname{act}(a, s)}{\longrightarrow} \lambda_{\text {eff }(a, s)}\left(x^{\prime}\right)} \operatorname{act}(a, s) \neq \delta, \psi, \chi \notin[\mathrm{F}] \\
& \frac{\mathrm{s}(x)=\phi}{\mathrm{s}\left(\lambda_{s}(x)\right)=\phi \wedge \psi} \operatorname{sig}(s)=\psi
\end{aligned}
$$

The state operator $\lambda_{s}$ allows, given the above-mentioned functions, processes to be executed in a state. Let $p$ be a closed term of sort $\mathbf{P}$. Then $\lambda_{s}(p)$ is the process $p$ executed in state $s$. The function act gives, for each action $a$ and state $s$, the action that results from executing $a$ in state $s$. The function eff gives, for each action $a$ and state $s$, the state that results from executing $a$ in state $s$. The function sig gives, for each state $s$, the proposition that holds at the start of any process executed in state $s$.

The additional axioms for $\lambda_{s}$, where $s \in S$, are given in Table[7. In this table, $a$ stands for an arbitrary constant from $\mathrm{A} \cup\{\delta\}$ and $\phi$ stands for an arbitrary closed term of sort B. SO1-SO5 have been taken from [8].

The following equations are among the equations derivable from the axioms of $\mathrm{ACP}_{\mathrm{ps}}^{\mathrm{ct}}+\mathrm{SO}$ :

$$
\lambda_{s}(\perp)=\perp, \quad \lambda_{s}(\delta)=\operatorname{sig}(s) \wedge \delta .
$$

All closed $\mathrm{ACP}_{\mathrm{ps}}^{\mathrm{ct}}+\mathrm{SO}$ terms of sort $\mathbf{P}$ can be reduced to a basic term.

Proposition 6 (Elimination). For all $\mathrm{ACP}_{\mathrm{ps}}^{\mathrm{ct}}+\mathrm{SO}$ closed terms $p$ of sort $\mathbf{P}$, there exists a $q \in \mathcal{B}$ such that $p=q$ is derivable from the axioms of $\mathrm{ACP}_{\mathrm{ps}}^{\mathrm{ct}}+\mathrm{SO}$.

Proof. The proof goes along the same line as the proof of Proposition 2

The additional transition rules for the state operators are given in Table 8. In this table, $a$ stands for an arbitrary constant from $A \cup\{\delta\}$ and $\phi$ stands for an arbitrary closed term of sort $\mathbf{B}$.

Bisimulation equivalence is a congruence with respect to the operators of $\mathrm{ACP}_{\mathrm{ps}}^{\mathrm{ct}}+\mathrm{SO}$. 
Proposition 7 (Congruence). For all closed $\mathrm{ACP}_{\mathrm{ps}}^{\mathrm{ct}}+\mathrm{SO}$ terms $p, q, p^{\prime}, q^{\prime}$ of

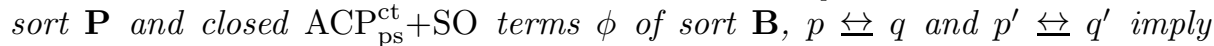

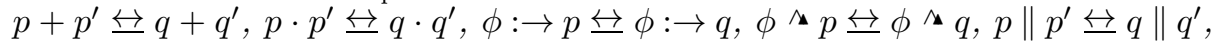

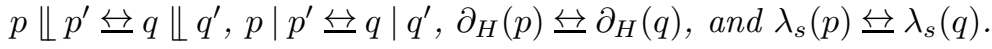

Proof. The proof goes along the same line as the proof of Proposition 3

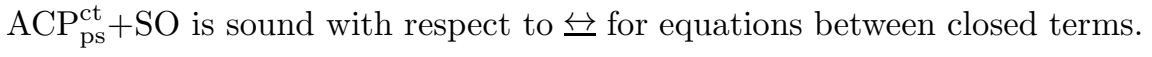

Theorem 5 (Soundness). For all closed $\mathrm{ACP}_{\mathrm{ps}}^{\mathrm{ct}}+\mathrm{SO}$ terms $p, q$ of sort $\mathbf{P}$,

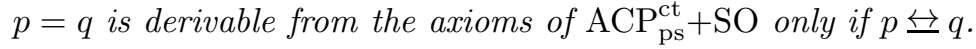

Proof. The proof goes along the same line as the proof of Theorem 3 ,

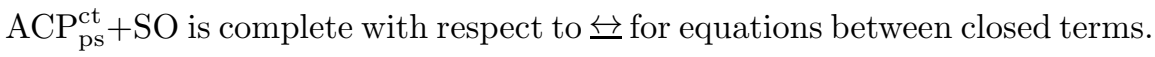

Theorem 6 (Completeness). For all closed $\mathrm{ACP}_{\mathrm{ps}}^{\mathrm{ct}}+\mathrm{SO}$ terms $p, q$ of sort $\mathbf{P}$,

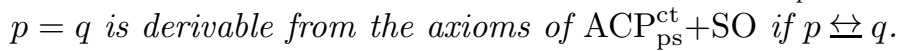

Proof. The proof goes along the same line as the proof of Theorem 4 ,

\section{Guarded Recursion}

In order to allow for the description of processes without a finite upper bound to the number of actions that it can perform, we add in this section guarded recursion to $\mathrm{ACP}_{\mathrm{ps}}^{\mathrm{ct}}$ and $\mathrm{ACP}_{\mathrm{ps}}^{\mathrm{ct}}+\mathrm{SO}$. The resulting theories are called $\mathrm{ACP}_{\mathrm{ps}}^{\mathrm{ct}}+\mathrm{REC}$ and $\mathrm{ACP}_{\mathrm{ps}}^{\mathrm{ct}}+\mathrm{SO}+\mathrm{REC}$, respectively.

A recursive specification over $\mathrm{ACP}_{\mathrm{ps}}^{\mathrm{ct}}$ is a set of recursion equations $E=$ $\left\{X=t_{X} \mid X \in V\right\}$ where $V$ is a set of variables of sort $\mathbf{P}$ and each $t_{X}$ is a term of sort $\mathbf{P}$ that only contains variables from $V$. We write $\mathrm{V}(E)$ for the set of all variables that occur on the left-hand side of an equation in E. A solution of a recursive specification $E$ is a set of processes (in some model of $\mathrm{ACP}_{\mathrm{ps}}^{\mathrm{ct}}$ ) $\left\{P_{X} \mid X \in \mathrm{V}(E)\right\}$ such that the equations of $E$ hold if, for all $X \in \mathrm{V}(E), X$ stands for $P_{X}$.

Let $t$ be a $\mathrm{ACP}_{\mathrm{ps}}^{\mathrm{ct}}$ term of sort $\mathbf{P}$ containing a variable $X$. We call an occurrence of $X$ in $t$ guarded if $t$ has a subterm of the form $a \cdot t^{\prime}$, where $a \in \mathrm{A}$, with $t^{\prime}$ containing this occurrence of $X$. A recursive specification $E$ over $\mathrm{ACP}_{\mathrm{ps}}^{\mathrm{ct}}$ is called a guarded recursive specification if all occurrences of variables in the right-hand sides of its equations are guarded or it can be rewritten to such a recursive specification using the axioms of $\mathrm{ACP}_{\mathrm{ps}}^{\mathrm{ct}}$ in either direction and/or the equations in $E$ from left to right. We are only interested in a model of $\mathrm{ACP}_{\mathrm{ps}}^{\mathrm{ct}}$ in which guarded recursive specifications have unique solutions.

For each guarded recursive specification $E$ over $\mathrm{ACP}_{\mathrm{ps}}^{\mathrm{ct}}$ and each variable $X \in \mathrm{V}(E)$, we add a constant of sort $\mathbf{P}$, standing for the unique solution of $E$ for $X$, to the constants of $\mathrm{ACP}_{\mathrm{ps}}^{\mathrm{ct}}$. This constant is denoted by $\langle X \mid E\rangle$. 
Table 9. Axioms for guarded recursion

\begin{tabular}{lll}
\hline$\langle X \mid E\rangle=\left\langle t_{X} \mid E\right\rangle$ & if $X=t_{X} \in E$ & $\mathrm{RDP}$ \\
$E \rightarrow X=\langle X \mid E\rangle$ & if $X \in \mathrm{V}(E)$ & $\mathrm{RSP}$ \\
\hline
\end{tabular}

Table 10. Transition rules for guarded recursion

\begin{tabular}{ll}
$\stackrel{\frac{\left\langle t_{X} \mid E\right\rangle \stackrel{\{\phi\} a}{\longrightarrow} \sqrt{ }}{\langle X \mid E\rangle \stackrel{\{\phi\} a}{\longrightarrow} \sqrt{ }} X=t_{X} \in E}{\frac{\left\langle t_{X} \mid E\right\rangle \stackrel{\{\phi\} a}{\longrightarrow} x^{\prime}}{\langle X \mid E\rangle \stackrel{\{\phi\} a}{\longrightarrow} x^{\prime}} X=t_{X} \in E}$ \\
$\frac{\mathbf{s}\left(\left\langle t_{X} \mid E\right\rangle\right)=\phi}{\mathbf{s}(\langle X \mid E\rangle)=\phi} X=t_{X} \in E$ \\
\hline
\end{tabular}

We will use the following notation. Let $t$ be a $\mathrm{ACP}_{\mathrm{ps}}^{\mathrm{ct}}$ term of sort $\mathbf{P}$ and $E$ be a guarded recursive specification over $\mathrm{ACP}_{\mathrm{ps}}^{\mathrm{ct}}$. Then we write $\langle t \mid E\rangle$ for $t$ with, for all $X \in \mathrm{V}(E)$, all occurrences of $X$ in $t$ replaced by $\langle X \mid E\rangle$.

The additional axioms for guarded recursion are the equations given in Table 9. In this table, $X, t_{X}$, and $E$ stand for an arbitrary variable of sort $\mathbf{P}$, an arbitrary $\mathrm{ACP}_{\mathrm{ps}}^{\mathrm{ct}}$ term, and an arbitrary guarded recursive specification over $\mathrm{ACP}_{\mathrm{ps}}^{\mathrm{ct}}$, respectively. Side conditions are added to restrict the variables, terms and guarded recursive specifications for which $X, t_{X}$ and $E$ stand. The additional axioms for guarded recursion are known as the recursive definition principle (RDP) and the recursive specification principle (RSP). The equations $\langle X \mid E\rangle=\left\langle t_{X} \mid E\right\rangle$ for a fixed $E$ express that the constants $\langle X \mid E\rangle$ make up a solution of $E$. The conditional equations $E \rightarrow X=\langle X \mid E\rangle$ express that this solution is the only one.

The additional transition rules for the constants $\langle X \mid E\rangle$ are given in Table10. In this table, $X, t_{X}$ and $E$ stand for an arbitrary variable of sort $\mathbf{P}$, an arbitrary $\mathrm{ACP}_{\mathrm{ps}}^{\mathrm{ct}}$ term and an arbitrary guarded recursive specification over $\mathrm{ACP}_{\mathrm{ps}}^{\mathrm{ct}}$, respectively.

Bisimulation equivalence is a congruence with respect to the operators of $\mathrm{ACP}_{\mathrm{ps}}^{\mathrm{ct}}+\mathrm{REC}$.

Proposition 8 (Congruence). For all closed $\mathrm{ACP}_{\mathrm{ps}}^{\mathrm{ct}}+\mathrm{REC}$ terms $p, q, p^{\prime}, q^{\prime}$ of

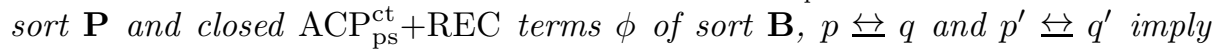

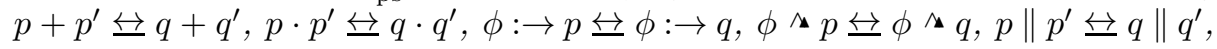

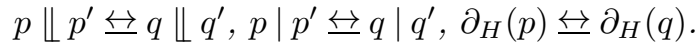

Proof. The proof goes along the same line as the proof of Proposition 3 .

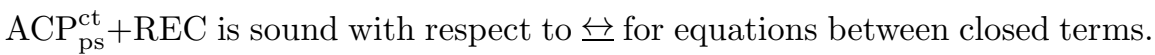

Theorem 7 (Soundness). For all closed $\mathrm{ACP}_{\mathrm{ps}}^{\mathrm{ct}}+\mathrm{REC}$ terms $p, q$ of sort $\mathbf{P}$,

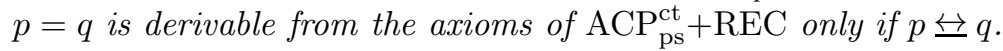


Proof. Because of Proposition 8 it is sufficient to prove the theorem for all closed $\mathrm{ACP}_{\mathrm{ps}}^{\mathrm{ct}}+\mathrm{REC}$ terms $p$ and $q$ for which $p=q$ is a closed substitution instance of an axiom of $\mathrm{ACP}_{\mathrm{ps}}^{\mathrm{ct}}+\mathrm{REC}$. With the exception of the closed substitution instances of RSP, the proof goes along the same line as the proof of Theorem 3 The proof of the validity of RSP is rather involved. We confine ourselves to a very brief outline of the proof. The transition rules for $\mathrm{ACP}_{\mathrm{ps}}^{\mathrm{ct}}+\mathrm{REC}$ determines a transition system for each process that can be denoted by a closed $\mathrm{ACP}_{\mathrm{ps}}^{\mathrm{ct}}+\mathrm{REC}$ term of sort $\mathbf{P}$. A model of $\mathrm{ACP}_{\mathrm{ps}}^{\mathrm{ct}}+\mathrm{REC}$ based on these transition systems can be constructed along the same line as the models of a generalization of ACPps constructed in [15]. An equation $p=q$ between closed $\mathrm{ACP}_{\mathrm{ps}}^{\mathrm{ct}}+\mathrm{REC}$ terms holds

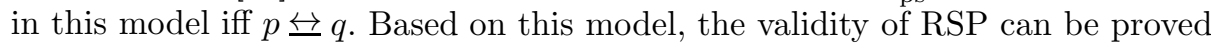
along the same line as in the proof of Theorem 10 from [15. The underlying ideas of that proof originate largely from $[9]$.

Guarded recursion can be added to $\mathrm{ACP}_{\mathrm{ps}}^{\mathrm{ct}}+\mathrm{SO}$ in the same way as it is added to $\mathrm{ACP}_{\mathrm{ps}}^{\mathrm{ct}}$ above, resulting in $\mathrm{ACP}_{\mathrm{ps}}^{\mathrm{ct}}+\mathrm{SO}+\mathrm{REC}$. It is easy to see that the above results, i.e. Proposition 8 and Theorem 7 go through for $\mathrm{ACP}_{\mathrm{ps}}^{\mathrm{ct}}+\mathrm{SO}+\mathrm{REC}$.

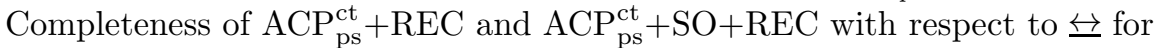
equations between closed terms can be obtained by restriction to the finite linear recursive specifications, i.e. the guarded recursive specifications with finitely many recursion equations where the right-hand side of each recursion equation can be written in the form $\chi \wedge \delta+\sum_{i \in\{1, \ldots, n\}} \phi_{i}: \rightarrow a_{i} \cdot X_{i}+\sum_{j \in\{1, \ldots, m\}} \psi_{j}: \rightarrow b_{j}$, where $n, m \in \mathbb{N}$, where $\chi \notin[\mathrm{F}]$, where $\phi_{i} \notin[\mathrm{F}], a_{i} \in \mathrm{A}$, and $X_{i}$ is variable of sort $\mathbf{P}$ for all $i \in\{1, \ldots, n\}$, and where $\psi_{j} \notin[\mathrm{F}]$ and $b_{j} \in \mathrm{A}$ for all $j \in\{1, \ldots, m\}$.

\section{Concluding Remarks}

We have presented $\mathrm{ACP}_{\mathrm{ps}}^{\mathrm{ct}}$, a version of $\mathrm{ACP} p$ s built on a paraconsistent propositional logic called $\mathrm{LP}^{\mathrm{J}, \mathrm{F}}$. $\mathrm{ACP}_{\mathrm{ps}}^{\mathrm{ct}}$ deals with processes with possibly selfcontradictory states by means of this paraconsistent logic. To our knowledge, processes with possibly self-contradictory states have not been dealt with in any theory or model of processes. This leaves nothing to be said about related work. However, it is worth mentioning that the need for a theory or model of processes with possibly self-contradictory states was already expressed in 24 .

In order to streamline the presentation of $\mathrm{ACP}_{\mathrm{ps}}^{\mathrm{ct}}$, we have left out the terminal signal emission operator, the global signal emission operator, and the root signal operator of ACPps and also the additional operators introduced in [8] other than the state operators. To our knowledge, these are exactly the operators that have not been used in any work based on ACPps. The root signal operator is an auxiliary operator which can be dispensed with and the global signal emission operator is an auxiliary operator which can be dispensed with in the absence of the terminal signal emission operator. The terminal signal emission operator makes it possible to express that a proposition holds at the termination of a process. 
$\mathrm{ACP}_{\mathrm{ps}}^{\mathrm{ct}}$ is a contradiction-tolerant version of ACPps [8. ACPps itself can be viewed as a simplification and specialization of ACPS [7. The simplification consists of the use of conditions instead of special actions to observe signals. The specialization consists of the use of the set of all propositions with propositional variables from a given set instead of an arbitrary free Boolean algebra over a given set of generators. Later, the generalization of ACPps to arbitrary such Boolean algebras has been treated in [15]. Moreover, a timed version of ACPps has been used in [14 as the basis of a process algebra for hybrid systems and a timed version of ACPps has been used in [17] to give a semantics to a specification language that was widely used in telecommunications at the time.

Timed versions of $\mathrm{ACP}_{\mathrm{ps}}^{\mathrm{ct}}$ may be useful in various applications. We believe that they can be obtained by combining $\mathrm{ACP}_{\mathrm{ps}}^{\mathrm{ct}}$ with a timed version of $\mathrm{ACP}$, such as $\mathrm{ACP}^{\mathrm{drt}}$ or $\mathrm{ACP}^{\text {srt }}$ from [10, in much the same way as timed versions of $\mathrm{ACPps}$ have been obtained in [14]17. Because idling of processes is taken into account, two forms of the guarded command operator can be distinguished in these timed versions, namely a non-waiting form and a waiting form (see e.g. [17]). A version of $\mathrm{ACP}_{\mathrm{ps}}^{\mathrm{ct}}$ with abstraction features like in $\mathrm{ACP}^{\tau}$ (see e.g. [11]) may be useful in

various applications as well. Working out a timed version of $\mathrm{ACP}_{\mathrm{ps}}^{\mathrm{ct}}$ and working out a version of $\mathrm{ACP}_{\mathrm{ps}}^{\mathrm{ct}}$ with abstraction features are options for further work. It is very important that case studies are carried out in conjunction with the theoretical work just mentioned to assess the degree of usefulness in practical applications.

$\mathrm{LP}^{\supset, \mathrm{F}}$ is Blok-Pigozzi algebraizable. However, although there must exist one, a conditional-equational axiomatization of the algebras concerned has not yet been devised. Owing to this, the equations derivable in $\mathrm{ACP}_{\mathrm{ps}}^{\mathrm{ct}}$ cannot always be derived by equational reasoning only. Another option for further work is devising the axiomatization referred to.

\section{Acknowledgements}

We thank two anonymous referees for carefully reading a preliminary version of this paper and for suggesting improvements of the presentation of the paper.

\section{References}

1. Arieli, O., Avron, A.: Three-valued paraconsistent propositional logics. In: Beziau, J.Y., Chakraborty, M., Dutta, S. (eds.) New Directions in Paraconsistent Logic. Springer Proceedings in Mathematics \& Statistics, vol. 152, pp. 91-129. SpringerVerlag (2015), http://dx.doi.org/10.1007/978-81-322-2719-9_4

2. Arieli, O., Avron, A., Zamansky, A.: Ideal paraconsistent logics. Studia Logica 99(1-3), 31-60 (2011), http://dx.doi.org/10.1007/s11225-011-9346-y

3. Arieli, O., Avron, A., Zamansky, A.: Maximal and premaximal paraconsistency in the framework of three-valued semantics. Studia Logica 97(1), 31-60 (2011), http://dx.doi.org/10.1007/s11225-010-9296-9

4. Avron, A.: Natural 3-valued logics - characterization and proof theory. The Journal of Symbolic Logic 56(1), 276-294 (1991), http://dx.doi.org/10.2307/2274919 
5. Avron, A.: On the expressive power of three-valued and four-valued languages. The Journal of Logic and Computation 9(6), 977-994 (1999), http://dx.doi.org/10.1093/logcom/9.6.977

6. Baeten, J.C.M., Bergstra, J.A.: Global renaming operators in concrete process algebra. Information and Control 78(3), 205-245 (1988), http://dx.doi.org/10.1016/0890-5401(88)90027-2

7. Baeten, J.C.M., Bergstra, J.A.: Process algebra with signals and conditions. In: Broy, M. (ed.) Programming and Mathematical Methods. NATO ASI Series, vol. F88, pp. 273-323. Springer-Verlag, Berlin (1992), http://dx.doi.org/10.1007/978-3-642-77572-7_13

8. Baeten, J.C.M., Bergstra, J.A.: Process algebra with propositional signals. Theoretical Computer Science 177(2), 381-405 (1997), http://dx.doi.org/10.1016/S0304-3975(96)00253-8

9. Baeten, J.C.M., Bergstra, J.A., Klop, J.W.: On the consistency of Koomen's fair abstraction rule. Theoretical Computer Science 51(1-2), 129-176 (1987), http://dx.doi.org/10.1016/0304-3975(87)90052-1

10. Baeten, J.C.M., Middelburg, C.A.: Process Algebra with Timing. Monographs in Theoretical Computer Science, An EATCS Series, Springer-Verlag, Berlin (2002), http://dx.doi.org/10.1007/978-3-662-04995-2

11. Baeten, J.C.M., Weijland, W.P.: Process Algebra, Cambridge Tracts in Theoretical Computer Science, vol. 18. Cambridge University Press, Cambridge (1990), http://dx.doi.org/10.1017/CBO9780511624193

12. Batens, D., de Clercq, K.: A rich paraconsistent extension of full positive logic. Logique et Analyse 185-188, 220-257 (2004), http://logica.ugent.be/ dirk/cluns_fin.pdf

13. Bergstra, J.A., Klop, J.W.: Algebra of communicating processes with abstraction. Theoretical Computer Science 37, 77-121 (1985), http://dx.doi.org/10.1016/0304-3975(85)90088-X

14. Bergstra, J.A., Middelburg, C.A.: Process algebra for hybrid systems. Theoretical Computer Science 335(2-3), 215-280 (2005), http://dx.doi.org/10.1016/j.tcs.2004.04.019

15. Bergstra, J.A., Middelburg, C.A.: Splitting bisimulations and retrospective conditions. Information and Computation 204(7), 1083-1138 (2006), http://dx.doi.org/10.1016/j.ic.2006.03.003

16. Bergstra, J.A., Middelburg, C.A.: Preferential choice and coordination conditions. Journal of Logic and Algebraic Programming 70(2), 172-200 (2007), http://dx.doi.org/10.1016/j.jlap.2006.08.004

17. Bergstra, J.A., Middelburg, C.A., Usenko, Y.S.: Discrete time process algebra and the semantics of SDL. In: Bergstra, J.A., Ponse, A., Smolka, S.A. (eds.) Handbook of Process Algebra, pp. 1209-1268. Elsevier, Amsterdam (2001), http://dx.doi.org/10.1016/B978-044482830-9/50036-9

18. Blok, W.J., Pigozzi, D.: Algebraizable Logics. No. 396 in Memoirs of the American Mathematical Society, American Mathematical Society, Providence (1989), http://dx.doi.org/10.1090/memo/0396

19. Carnielli, W.A., Coniglio, M.E., Marcos, J.: Logics of formal inconsistency. In: Gabbay, D., Guenthner, F. (eds.) Handbook of Philosophical Logic, vol. 14, pp. 193. Springer-Verlag, Berlin (2007), http://dx.doi.org/10.1007/978-1-4020-6324-4_1

20. D'Ottaviano, I.M.L.: The completeness and compactness of a three-valued first-order logic. Revista Colombiana de Matemáticas 19, 77-94 (1985), https://eudml.org/doc/181748 
21. Fokkink, W.J., van Glabbeek, R.J.: Ntyft/ntyxt rules reduce to ntree rules. Information and Computation 126(1), 1-10 (1996), http://dx.doi.org/10.1006/inco.1996.0030

22. Fokkink, W.J., Verhoef, C.: A conservative look at operational semantics with variable binding. Information and Computation 146(1), 24-54 (1998), http://dx.doi.org/10.1006/inco.1998.2729

23. Greenfield, P., Kuo, D., Nepal, S., Fekete, A.: Consistency for web services applications. In: Böhm, K., et al. (eds.) VLDB 2005. VLDB Conferences, vol. 31, pp. 1199-1203. VLDB Endowment (2005), http://www.vldb2005.org/program/paper/thu/p1199-greenfield.pdf

24. Hewitt, C.: ORGs for scalable, robust, privacy-friendly client cloud computing. IEEE Internet Computing 12(5), 96-99 (2008), http://dx.doi.org/10.1109/MIC.2008.107

25. Hoare, C.A.R.: Communicating Sequential Processes. Prentice-Hall, Englewood Cliffs (1985)

26. Middelburg, C.A.: A survey of paraconsistent logics (2011), arXiv:1103.4324[cs.LO]

27. Milner, R.: Communication and Concurrency. Prentice-Hall, Englewood Cliffs (1989)

28. Priest, G.: The logic of paradox. Journal of Philosophical Logic 8(1), 219-241 (1979), http://dx.doi.org/10.1007/BF00258428

29. Py, F., Ingrand, F.: Dependable execution control for autonomous robots. In: IROS 2004. pp. 1136-1141. IEEE (2004), http://dx.doi.org/10.1109/IROS.2004.1389549

30. Qu, H., Veres, S.M.: On efficient consistency checks by robots. In: ECC 2014. pp. 336-343. IEEE (2014), http://dx.doi.org/10.1109/ECC.2014.6862528

31. Verhoef, C.: A general conservative extension theorem in process algebra. In: Olderog, E.R. (ed.) PROCOMET '94. pp. 149-168. Elsevier, Amsterdam (1994) 